\title{
Optimal Control of Sliding Droplets using the Contact Angle Distribution *
}

\author{
Henning Bonart ${ }^{1}$ and Christian Kahle ${ }^{2, *}$ \\ ${ }^{1}$ Technische Universität Berlin, Process Dynamics and Operations Group, \\ Straße des 17. Juni 135, 10623 Berlin, Germany \\ ${ }^{2}$ Universität Koblenz-Landau, Campus Koblenz, \\ Universitätsstraße 1, 56070 Koblenz, Germany \\ ${ }^{*}$ Corresponding author: kahle@uni-koblenz.de (Christian Kahle)
}

February 10, 2020

\begin{abstract}
Controlling the shape and position of moving and pinned droplets on a solid surface is an important feature often found in microfluidic applications. In this work, we consider a well investigated phase field model including contact line dynamics as the state system for an (open-loop) optimal control problem. Here the spatially and temporally changeable contact angles between droplet and solid are considered as the control variables. We consider a suitable, energy stable, time discrete version of the state equation in our optimal control problem. We discuss regularity of the solution to the time discrete state equation and its continuity and differentiability properties. Furthermore, we show existence of solutions and state first order optimality conditions to the optimal control problem. We illustrate our results by actively pushing a droplet uphill against gravity in an optimal way.
\end{abstract}

\section{Introduction}

Controlling the shape and position of moving and pinned droplets on a solid surface is an important feature often found in microfluidics applications. On a lab-on-a-chip droplets can be transported across the solid surface by a contact angle gradient, and merged, split or mixed in a controlled fashion [51]. Thereby, the shape of the droplets influences the heat and mass exchanged with the solid surface and the surrounding fluid phase [6]. Furthermore, the distribution of nutritiens and the direction of growth of bio-films and cell cultures depends on the shape and the surface structure, too [23]. In optical applications, liquid droplets can act as flexible lenses with continuous refraction index ranges. The curvature and hence their focal length can be tuned by adjusting their shape through the contact angle distribution [44].

${ }^{*}$ The first author acknowledges the German Research Foundation (DFG) for the financial support within the project RE 1705/16-1. 
In all these processes and applications the shape and position of the droplet (or gas-liquid interface) plays a significant role. However, automating them, e.g., for high-throughput applications, does rarely involve model-based optimal control strategies. The potential of optimal control for microfluidics is for example shown in the following publications. In [47] the control of the footprint and shape of a static droplet is presented. The position of a moving droplet and its shape without the influence of gravity is considered in [13]. Results on the position of the gas-liquid interface of rising liquid in a capillary are provided in [25].

In this work, we are concerned with the optimal control of droplets where the static contact angle between solid surface and droplet serves as the control variable. In Figure1, a general physical setting of the problem is illustrated. Initially, a liquid droplet is placed on an inclined solid surface (dark gray). Due to gravity $g$, the droplet slides down the surface. However, with the help of the patches $u_{1}$ to $u_{4}$ we control the contact angles $\theta_{1}$ and $\theta_{2}$ between droplet and solid. Depending on the actual objective, we use this control e.g. to track a desired shape (dashed) over a given time horizon. In this way, we are able to impose the desired shape and position at a specific time (light gray). As observed in the very famous experiments by Chaudhury and Whitesides [18], we are even able to push the droplet uphill against gravity. Note, that in practical applications, the control patches represent electrodes and the contact angle is varied using an electric potential (socalled electrowetting, see [49]). For details on the physical background as well as the technical implementation in devices we refer to [49].

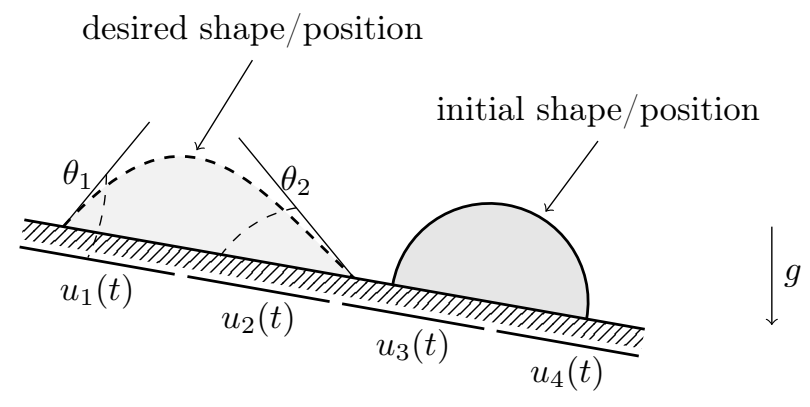

Figure 1: Physical setting of the optimal control problem.

This paper is organized as follows. In Section 2 we introduce the model for the moving contact line dynamics and review some properties. Thereafter we introduce a time discrete approximation of this model in Section 3 and investigate the regularity properties of the resulting equations. Moreover, we show continuity and differentiability properties that are necessary in the subsequent section. In Section 4 we introduce an optimization problem, that models the control of a droplet by the contact angle distribution. Using the results from Section 3 we show existence of solutions and derive first order optimality conditions. Finally, in Section 5 we illustrate our results by optimally pushing a droplet uphill.

\section{The model for moving contact line dynamics}

In this section we introduce the mathematical model under consideration. It is a Cahn-Hilliard Navier-Stokes system with a moving contact line model for the Cahn-Hilliard system and no-slip 
boundary data for the Navier-Stokes system, see for example [1, 4, 33, 52].

The model consists of a velocity field $v(t)$ and a pressure field $p(t)$ that satisfy the NavierStokes-type equation (12)-(2) and a phase field $\varphi(t)$ and a chemical potential $\mu(t)$ that satisfy the advective Cahn-Hilliard equation (3)-(4). In continuous and strong setting the model reads as follows.

Let $\Omega \subset \mathbb{R}^{n}, n \in\{2,3\}$ denote a polynomially/polygonally bounded Lipschitz domain with boundary $\partial \Omega$ and with unit outer normal $\nu_{\Omega}$. Let $(0, T)$ denote a time interval. Given sufficient smooth initial data $v_{0}$, and $\varphi_{0}$ find $v(t, x), p(t, x), \varphi(t, x)$, and $\mu(t, x)$ such that for almost all $t \in(0, I]$ it holds

$$
\begin{aligned}
\rho \partial_{t} v+((\rho v+J) \cdot \nabla) v+R \frac{v}{2}-\operatorname{div}(2 \eta D v)+\nabla p & =-\varphi \nabla \mu+\rho g & & \text { in } \Omega, \\
-\operatorname{div}(v) & =0 & & \text { in } \Omega, \\
\partial_{t} \varphi+v \cdot \nabla \varphi-b \Delta \mu & =0 & & \text { in } \Omega, \\
-c_{W} \sigma_{l g} \epsilon \Delta \varphi+c_{W} \sigma_{l g} \epsilon^{-1} W^{\prime}(\varphi) & =\mu & & \text { in } \Omega, \\
v & =0 & & \text { on } \partial \Omega, \\
r \partial_{t} \varphi+L(\varphi) & =0 & & \text { on } \partial \Omega, \\
\nabla \mu \cdot \nu_{\Omega} & =0 & & \text { on } \partial \Omega,
\end{aligned}
$$

where we set $J:=-b \frac{d \rho}{d \varphi} \nabla \mu, R:=-b \nabla \frac{d \rho}{d \varphi} \cdot \nabla \mu, 2 D v:=\nabla v+(\nabla v)^{t}$, and $L:=c_{W} \sigma_{l g} \epsilon \nabla \varphi \cdot \nu_{\Omega}+\gamma_{u}^{\prime}(\varphi)$.

The (nonlinear) functions $\rho(\varphi)$ and $\eta(\varphi)$ denote the density and the viscosity of the fluid, respectively. See Remark 1 for further discussion of $\rho$ and $\eta$. We note, that the additional term $R$ in (11) appears from the nonlinearity of $\rho$ and vanishes in case of a linear function $\rho(\varphi)$, see [1]. The gravitational acceleration is denoted by $g$, while $b>0$ denotes the mobility of the fluid, that for simplicity is taken as constant. The constant $\sigma_{l g}$ denotes the surface tension at the fluidic interface between the two phases. The interface is considered as diffuse with a width proportional to $\epsilon$. The function $W(\varphi)$ denotes a dimensionless potential of double-well type, with two strict minima at \pm 1 that define the pure phases. See Remark 2 for further discussion of $W$. The constant $c_{W}$ is also defined in Remark 2. In the following it is convenient to call the phase that is defined by $\varphi \equiv-1$ as gas and the phase that is defined by $\varphi \equiv 1$ as liquid. The material outside of $\Omega$ is called solid. Finally, $\gamma_{u}(\varphi)$ denotes the contact line energy and is further explained in Remark 3 . The constant $r \geq 0$ denotes a phenomenological parameter allowing for nonequilibrium contact angles at the contact line.

Existence of a solution to (1)-(7) without the additional term $R$ is shown in 33 and several numerical schemes are tested. Concerning further analytical results for the bulk model with homogeneous boundary data we refer to [1-4, 32]. For results on phase field models, that contain a contact line model, we refer to [20, 26, 60].

Concerning numerical schemes for (11)-(17) and submodels thereof, we refer to [7, 8, 19, 27, 28, 33 37, 53, 54, 59, 61, 62].

For results on control and boundary control of the Cahn-Hilliard and Cahn-Hilliard NavierStokes equation we refer to [21, 24, 29, 30, 39, 41, 42, 46].

Remark 1 (Nonlinear density and viscosity). Note that in general there is no quantitative upper bound available for $\varphi$ and thus in particular $|\varphi|>1$ is commonly observed. Thus a linear relation between $\varphi$ and $\rho$ might lead to negative densities and viscosities in practice. This especially appears 


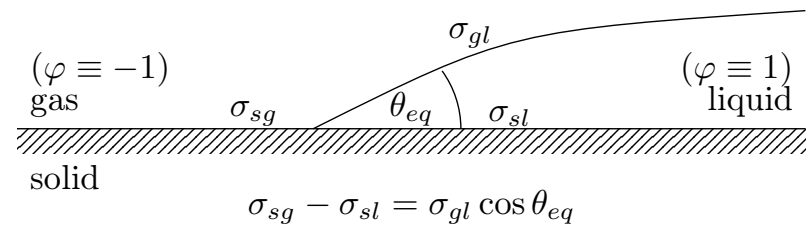

Figure 2: Definition of parameters in Young's law.

for large density ratios, compare, e.g., [33, Rem. 4.1]. Therefore we use the modification proposed in [1] and add the term $R$ in (11) that compensates for a nonlinear density. We also refer to, e.g., [32, Rem. 2.1], [17, Rem. 1], and [28, Rem. 6] for further discussions of this topic. In the following we assume that $\rho$ and $\eta$ are bounded, strictly positive, continuously differentiable and globally Lipschitz continuous. Additionally we require $\rho \in C^{2}(\mathbb{R})$ and define $\rho_{\min }:=\min _{t \in \mathbb{R}} \rho(t)$. Moreover $\rho(1)=\rho_{l}$, $\rho(-1)=\rho_{g}, \eta(1)=\eta_{l}$, and $\eta(-1)=\eta_{g}$ is satisfied, where $\rho_{l / g}$ denotes the density of the gas and liquid phase, while $\eta_{l / g}$ denote the corresponding viscosity.

We note, that with a nonlinear density function, while $\int_{\Omega} \varphi \mathrm{dx}$ is a conserved quantity, the total mass $\int_{\Omega} \rho(\varphi) \mathrm{dx}$ is only conserved if $\rho(\varphi)$ is a linear function on the (a-priori unknown) image of $\varphi$, see e.g., [28, Rem. 1].

Remark 2 (The free energy potential). The free energy potential $W$ is a function with exactly two minima at \pm 1 with $W( \pm 1)=0$. Additonally we assume, that $W \in C^{2,1}(\mathbb{R})$ and that there exists a constant $C>0$ such that

$$
W(\varphi) \leq C\left(1+|\varphi|^{4}\right), \quad\left|W^{\prime}(\varphi)\right| \leq C\left(1+|\varphi|^{3}\right), \quad\left|W^{\prime \prime}(\varphi)\right| \leq C\left(1+|\varphi|^{2}\right) .
$$

The function $W$ further admitts a convex-concave splitting $W=W_{+}+W_{-}$such that $W_{+}$is a convex function with $W_{+}^{\prime}(0)=0$ and $W_{-}$is a concave function. The assumption (8) also holds for $W_{+}$ and $W_{-}$individually.

The parameter $c_{W}$ is defined as $c_{W}^{-1}:=\int_{-\infty}^{\infty} 2 W\left(\Phi_{0}(z)\right) \mathrm{dz}$, where $\Phi_{0}(z)$ denotes the first order approximation of $\varphi$ that satisfies $\Phi_{0}(z)_{z z}=W^{\prime}\left(\Phi_{0}(z)\right)$ see [4, Sec. 4.3.4].

We refer to, e.g., [17, Rem. 2] for further discussion of free energy potentials.

Remark 3 (The contact line energy). The contact line energy $\gamma_{u}$ is given by

$$
\gamma_{u}(\varphi):=\frac{\sigma_{s l}+\sigma_{s g}}{2}+\sigma_{l g}\left(\cos \left(\theta_{e q}\right)+B u\right) \vartheta(\varphi)
$$

Here $\sigma_{s l}$ denotes the physical surface tension between solid and liquid, $\sigma_{s g}$ denotes the physical surface tension between solid and gas, and $\sigma_{l g}$, as before, denotes the physical surface tension between liquid and gas. The equilibrium contact angle $\theta_{e q}$ between the interface and the solid is measured in the liquid phase. These variables satisfy Young's law, namely

$$
\sigma_{s l}-\sigma_{s g}=\sigma_{l g} \cos \theta_{e q}
$$

see Figure 2.

The function $\vartheta$ satisfies $\vartheta \in C^{1,1}(\mathbb{R}) \cap C^{2}(\mathbb{R})$ and there exists $L<\infty$ such that $\max _{s \in \mathbb{R}}\left|\gamma^{\prime \prime}(s)\right| \leq$ L. Further it holds $\vartheta(t)=-\frac{1}{2}$ for $t \leq-1$ and $\vartheta(t)=\frac{1}{2}$ for $t \geq 1$. A typical choice is $\vartheta(\varphi)=$ $\frac{1}{2} \sin \left(\frac{\pi}{2} \min (1, \max (-1, \varphi))\right)$. We refer to [17, Rem. 3] and the references therein for a discussion. 
The function $u \in U$ denotes a given control from some control space $U$, while $B: U \rightarrow$ $L^{2}\left(0, T ; L^{2}(\partial \Omega)\right)$ denotes a linear, continuous and injective control operator. The contact line model implies natural bounds for Bu and we thus define the convex and closed subset

$$
U_{a d}^{0}:=\left\{u \in U \mid-1 \leq \cos \left(\theta_{e q}\right)+B u \leq 1\right\} \subset U
$$

as largest set of admissable controls. Note that, since $B$ is injective, in fact $U_{a d}^{0}$ is a bounded subset.

Notation 4. We use the common notation for Sobolev and Hilbert spaces, see e.g. [5]. Especially by $L^{2}(\Omega)$ we denote the Hilbert space of square integrable functions on a domain $\Omega$. The norm and inner product in $L^{2}(\Omega)$ are denoted by $\|\cdot\|$ and $(\cdot, \cdot)$. The space $L^{\infty}(\Omega)$ denotes the space of essentially bounded functions, and the space $H^{1}(\Omega)$ denotes the space of square integrable functions, that admit a weak derivative that is square integrable.

Additionally to the standard notation, we introduce the space of weakly solenoidal functions

$$
H_{\sigma}(\Omega):=\left\{v \in H_{0}^{1}(\Omega)^{n} \mid(\operatorname{div} v, q)=0 \forall q \in L^{2}(\Omega), \int_{\Omega} q \mathrm{dx}=0\right\}
$$

Moreover, in the following we restrict to solenoidal velocity fields from $H_{\sigma}(\Omega)$ and thus skip the pressure in the equations.

For notational convenience in the following we set $\sigma=c_{W} \sigma_{l g}$.

\section{Analysis of the time discrete model}

For a practical implementation we introduce a time grid $0=t_{0}<t_{1}<\ldots<t_{m-1}<t_{m}<\ldots<$ $t_{M}=T$. For the sake of notational simplicity let the time grid be equidistant with step size $\tau>0$. We consider the following time discrete version of (17)-(7) in weak form.

Given $\varphi_{0} \in H^{1}(\Omega) \cap L^{\infty}(\Omega), v_{0} \in H_{\sigma}(\Omega)$, and $u \in U_{a d}^{0}$. Find sequences $v_{\tau}=\left(v^{m}\right)_{m=1}^{M} \in$ $\left(H_{\sigma}(\Omega)\right)^{M}, \varphi_{\tau}=\left(\varphi^{m}\right)_{m=1}^{M} \in\left(H^{1}(\Omega) \cap L^{\infty}(\Omega)\right)^{M}, \mu_{\tau}=\left(\mu^{m}\right)_{m=1}^{M} \in\left(W^{1, q}(\Omega)\right)^{M}, q>n$, such that for $m=1, \ldots, M$ and for all $w^{m} \in H_{\sigma}(\Omega), \Phi^{m} \in H^{1}(\Omega)$, and $\Psi^{m} \in H^{1}(\Omega)$ the following equations

$$
\begin{array}{r}
\frac{1}{\tau}\left(\frac{\rho^{m}+\rho^{m-1}}{2} v^{m}-\rho^{m-1} v^{m-1}, w^{m}\right)+a\left(\rho^{m-1} v^{m-1}+J^{m}, v^{m}, w^{m}\right)+\left(2 \eta^{m} D v^{m}, D w^{m}\right) \\
+\left(\varphi^{m-1} \nabla \mu^{m}, w^{m}\right)-\left(g \rho^{m}, w^{m}\right)=0 \\
\frac{1}{\tau}\left(\varphi^{m}-\varphi^{m-1}, \Psi^{m}\right)-\left(\varphi^{m-1} v^{m-1}, \nabla \Psi^{m}\right)+\frac{\tau}{\rho_{\min }}\left(\left|\varphi^{m-1}\right|^{2} \nabla \mu^{m}, \nabla \Psi^{m}\right)+b\left(\nabla \mu^{m}, \nabla \Psi^{m}\right)=0 \\
\sigma \epsilon\left(\nabla \varphi^{m}, \nabla \Phi^{m}\right)+\frac{\sigma}{\epsilon}\left(W_{+}^{\prime}\left(\varphi^{m}\right)+W_{-}^{\prime}\left(\varphi^{m-1}\right), \Phi^{m}\right)-\left(\mu^{m}, \Phi^{m}\right) \\
+r\left(B^{m}, \Phi^{m}\right)_{\partial \Omega}+\left(\frac{S_{\gamma}}{2}\left(\varphi^{m}-\varphi^{m-1}\right)+\gamma_{u}^{\prime}\left(\varphi^{m-1}\right), \Phi^{m}\right)_{\partial \Omega}
\end{array}
$$

with $J^{m}:=-b \rho^{\prime}\left(\varphi^{m}\right) \nabla \mu^{m}, B^{m}:=\left(\frac{\varphi^{m}-\varphi^{m-1}}{\tau}\right), \rho^{m}:=\rho\left(\varphi^{m}\right), \rho^{m-1}:=\rho\left(\varphi^{m-1}\right), \eta^{m}:=\eta\left(\varphi^{m}\right)$, and $\eta^{m-1}:=\eta\left(\varphi^{m-1}\right)$. For some $q>n$ (if $n=2$ ) or $q \geq n$ (if $n=3$ ) the trilinear form 
$a:\left(L^{q}(\Omega)\right)^{n} \times H^{1}(\Omega)^{n} \times H^{1}(\Omega)^{n}$ is defined by

$$
a(u, v, w):=\frac{1}{2} \int_{\Omega}((u \cdot \nabla) v) \cdot w-\frac{1}{2} \int_{\Omega}((u \cdot \nabla) w) \cdot v .
$$

The contact line energy $\gamma_{u}^{\prime}\left(\varphi^{m-1}\right)$ is given by $\gamma_{u}^{\prime}\left(\varphi^{m-1}\right)=\sigma_{l g}\left(\cos \left(\theta_{e q}\right)+B_{m} u\right) \vartheta^{\prime}\left(\varphi^{m-1}\right)$, with $B_{m} u:=\frac{1}{\tau} \int_{t_{m-1}}^{t_{m}}(B u)(t) \mathrm{dt}$. The parameter $S_{\gamma}$ is a stabilization parameter and satisfies $S_{\gamma} \geq$ $\frac{1}{2} \max _{s}\left|\gamma^{\prime \prime}(s)\right|$, see e.g. [8, 17].

We note, that if necessary we consider $v_{\tau}, \varphi_{\tau}$, and $\mu_{\tau}$ as piecewise constant functions in time to evaluate time depending norms. Here we set $\varphi_{\tau}(t) \equiv \varphi^{m}$ for $t \in\left(t_{m-1}, t_{m}\right]$, and correspondingly for $v_{\tau}$ and $\mu_{\tau}$.

Assumption 5. The regularity $\mu^{m} \in W^{1, q}(\Omega)$ with $q>n$ is required for the trilinear form $a(\cdot, \cdot, \cdot)$ to be well defined, since $\nabla \mu^{m}$ appears in the first argument of the trilinear form a. For $n=3$ this can be reduced to $\mu \in W^{1,3}(\Omega)$ and in the following we assume $n=3$.

Remark 6 (On the time discretizations of $W$ and $\gamma$ ). For the time discretization of $\gamma$ we use a linear stabilization scheme, to guarantee energy stability of the scheme, see Theorem $\mathbf{7}$. However, in the bulk domain, we use a convex-concave splitting for $W$ to guarantee energy stability.

We note, that the proposed linearization scheme for $\gamma_{u}$ might also be applied for $W$. Typically we obtain broader interfaces and a severe effect on the interface dynamic with this approach and therefor refrain from using this scheme for the bulk energy.

In [17] we use the convex-concave splitting scheme also for the contact line energy $\gamma_{u}$. In Section 4 we consider the term $B_{m} u$ in $\gamma_{u}$ as control variable and thus $\gamma_{u}^{\prime}$ in general does not have a uniform sign. Therefor we can not use a similar convex-concave splitting for the discretization of $\gamma_{u}$ in this work.

For further discretization schemes, we also refer to [17, 37, 53, 59, 61] and note, that typically the convex-concave splitting seems to be a very good compromise between stable interfaces and the validity of an energy inequality in the time discrete setting.

Theorem 7 (Energy inequality). Assume, that there exists a solution to (10)-(12). Then the following energy inequality holds on one time instance $m$.

$$
\begin{aligned}
& \frac{1}{2} \int_{\Omega} \rho^{m}\left|v^{m}\right|^{2}+\sigma \int_{\Omega} \frac{\epsilon}{2}\left|\nabla \varphi^{m}\right|^{2}+\frac{1}{\epsilon} W\left(\varphi^{m}\right)+\int_{\partial \Omega} \gamma\left(\varphi^{m}\right) \\
& +\tau\left(\int_{\Omega} 2 \eta^{m}\left|D v^{m}\right|^{2}+b \int_{\Omega}\left|\nabla \mu^{m}\right|^{2}+r \int_{\partial \Omega}\left|B^{m}\right|^{2}\right) \\
& \leq \frac{1}{2} \int_{\Omega} \rho^{m-1}\left|v^{m-1}\right|^{2}+\sigma \int_{\Omega} \frac{\epsilon}{2}\left|\nabla \varphi^{m-1}\right|^{2}+\frac{1}{\epsilon} W\left(\varphi^{m-1}\right)+\int_{\partial \Omega} \gamma\left(\varphi^{m-1}\right)+\tau \int_{\Omega} \rho^{m} g \cdot v^{m} .
\end{aligned}
$$

Moreover, by summing over (13) we obtain

$$
\begin{aligned}
& \frac{1}{2} \int_{\Omega} \rho^{m}\left|v^{m}\right|^{2}+\sigma \int_{\Omega} \frac{\epsilon}{2}\left|\nabla \varphi^{m}\right|^{2}+\frac{1}{\epsilon} W\left(\varphi^{m}\right)+\int_{\partial \Omega} \gamma\left(\varphi^{m}\right) \\
& +\tau \sum_{k=1}^{m}\left(\int_{\Omega} 2 \eta^{k}\left|D v^{k}\right|^{2}+b \int_{\Omega}\left|\nabla \mu^{k}\right|^{2}+r \int_{\partial \Omega}\left|B^{k}\right|^{2}\right) \\
& \leq \frac{1}{2} \int_{\Omega} \rho^{0}\left|v^{0}\right|^{2}+\sigma \int_{\Omega} \frac{\epsilon}{2}\left|\nabla \varphi^{0}\right|^{2}+\frac{1}{\epsilon} W\left(\varphi^{0}\right)+\int_{\partial \Omega} \gamma\left(\varphi^{0}\right)+\sum_{k=1}^{m} \int_{\Omega} \rho^{k} g \cdot v^{k} .
\end{aligned}
$$


Proof. The proof follows standard arguments and we refer to, e.g., [8, 17, 33].

Lemma 8. Let $\varphi^{m-1} \in H^{1}(\Omega) \cap L^{\infty}(\Omega)$ and $v^{m-1} \in H_{\sigma}(\Omega)$ be given. Moreover, let $u \in U_{a d}$ be satisfied. For every $m$, there exists exactly one solution to (10)-(12). Moreover, the following stability estimate holds:

$$
\begin{aligned}
\left\|v^{m}\right\|_{H^{1}(\Omega)} & +\left\|\varphi^{m}\right\|_{H^{1}(\Omega)}+\left\|\varphi^{m}\right\|_{C(\bar{\Omega})}+\left\|\mu^{m}\right\|_{W^{1,3}(\Omega)} \\
& \leq C\left(\left\|\varphi^{m-1}\right\|_{H^{1}(\Omega)},\left\|\varphi^{m-1}\right\|_{L^{\infty}(\Omega)},\left\|v^{m-1}\right\|_{H^{1}(\Omega)}\right),
\end{aligned}
$$

where the right hand side is a polynomial of its arguments. The result is not stable with respect to $\tau \rightarrow 0$ or $\epsilon \rightarrow 0$.

Proof. As systems (10) and (11)-(12) are decoupled, we first argue the existence for the latter system. The existence of a solution $\varphi^{m} \in H^{1}(\Omega), \mu^{m} \in H^{1}(\Omega)$ to (11)-(12) can be shown by considering a suitable minimization problem, compare, e.g., [38, 40]. This solution satisfies

$$
\left\|\varphi^{m}\right\|_{H^{1}(\Omega)}+\left\|\mu^{m}\right\|_{H^{1}(\Omega)} \leq C\left(\left\|\varphi^{m-1}\right\|_{H^{1}(\Omega)},\left\|\varphi^{m-1}\right\|_{L^{\infty}(\Omega)},\left\|v^{m-1}\right\|_{H^{1}(\Omega)}\right),
$$

where the right hand side is a polynomial of its arguments. The uniqueness then follows from assuming the existence of two solutions, and exploiting the monotonicity of $W_{+}^{\prime}$, see [28, Thm. 4].

Next we deduce the higher regularity for $\varphi^{m}$. Since $\varphi^{m} \in H^{1}(\Omega) \hookrightarrow L^{6}(\Omega)$ together with (8) it holds $W_{+}^{\prime}\left(\varphi^{m}\right) \in L^{2}(\Omega)$. Further, since $\gamma_{u}^{\prime}$ is Lipschitz continuous, we have from $\varphi^{m-1} \in H^{1 / 2}(\partial \Omega)$ that $\gamma_{u}^{\prime}\left(\varphi^{m-1}\right) \in H^{1 / 2}(\partial \Omega)$. Now we employ [56, Thm. 4.8] to observe that $\varphi^{m}$ is continuous and that

$$
\begin{aligned}
\left\|\varphi^{m}\right\|_{H^{1}(\Omega)}+\left\|\varphi^{m}\right\|_{C(\bar{\Omega})} & \leq C\left(\left\|\mu^{m}+W_{-}^{\prime}\left(\varphi^{m-1}\right)\right\|_{L^{2}(\Omega)}+\left\|\gamma_{u}^{\prime}\left(\varphi^{m-1}\right)\right\|_{H^{1 / 2}(\partial \Omega)}\right) \\
& \leq C\left(\left\|\mu^{m}\right\|_{L^{2}(\Omega)}+\left\|\varphi^{m-1}\right\|_{H^{1}(\Omega)}\right),
\end{aligned}
$$

where in the last line we use the properties of $W_{-}^{\prime}$ and the Lipschitz continuity of $\gamma_{u}^{\prime}$.

Now we deduce the higher regularity for $\mu^{m}$. Since $v^{m-1} \in H_{\sigma}(\Omega) \hookrightarrow L^{6}(\Omega)^{n}$, we have $\operatorname{div}\left(\varphi^{m-1} v^{m-1}\right) \in L^{3 / 2}(\Omega)$ and from [22, Thm. 1.9, Thm. 5.3] we thus have $\mu^{m} \in W^{1,3}(\Omega)$. Additionally we obtain

$$
\left\|\mu^{m}\right\|_{W^{1,3}(\Omega)} \leq C\left(\left\|\varphi^{m}\right\|_{L^{2}(\Omega)},\left\|\varphi^{m-1}\right\|_{H^{1}(\Omega)},\left\|\varphi^{m-1}\right\|_{L^{\infty}(\Omega)},\left\|v^{m-1}\right\|_{H^{1}(\Omega)}\right),
$$

where the right hand side is a polynomial of its arguments.

Finally, the existence of a unique solution $v^{m} \in H_{\sigma}(\Omega)$ to (10) readily follows by Lax-Milgram's theorem, and leads to

$$
\left\|v^{m}\right\|_{H^{1}(\Omega)} \leq C\left(\left\|\varphi^{m-1}\right\|_{H^{1}(\Omega)},\left\|\varphi^{m-1}\right\|_{L^{\infty}(\Omega)},\left\|\mu^{m}\right\|_{W^{1,3}(\Omega)},\left\|v^{m-1}\right\|_{H^{1}(\Omega)}\right),
$$

where the right hand side is a polynomial of its arguments. Here we used that $\rho$ and $\eta$ are bounded independent of their arguments.

Summing over (16), (18), and (19) and using (15) leads to the desired result.

Corollary 9. By iterating the result from Lemma 8 we bound the solution at any time instance by the initial data, namely

$$
\left\|v^{m}\right\|_{H^{1}(\Omega)}+\left\|\varphi^{m}\right\|_{H^{1}(\Omega)}+\left\|\varphi^{m}\right\|_{C(\bar{\Omega})}+\left\|\mu^{m}\right\|_{W^{1,3}(\Omega)} \leq C\left(\left\|\varphi^{0}\right\|_{H^{1}(\Omega)},\left\|\varphi^{0}\right\|_{L^{\infty}(\Omega)},\left\|v^{0}\right\|_{H^{1}(\Omega)}\right),
$$


for $m=1, \ldots, M$, or equivalently

$$
\left\|v_{\tau}\right\|_{l^{\infty}\left(H^{1}(\Omega)\right)}+\left\|\varphi_{\tau}\right\|_{l^{\infty}\left(H^{1}(\Omega)\right)}+\left\|\varphi_{\tau}\right\|_{l^{\infty}(C(\bar{\Omega}))}+\left\|\mu_{\tau}\right\|_{l^{\infty}\left(W^{1,3}(\Omega)\right)} \leq C\left(\left\|\varphi^{0}\right\|_{H^{1}(\Omega)},\left\|\varphi^{0}\right\|_{L^{\infty}(\Omega)},\left\|v^{0}\right\|_{H^{1}(\Omega)}\right) .
$$

Here the constant depends polynomially on its arguments.

Remark 10. We stress that the stability result from Lemma 8 is independent of the control $u$ and the static contact angle because we already use the stated bounds $-1 \leq \cos \left(\theta_{\text {eq }}\right)+B u \leq 1$ for $u \in U_{a d}^{0}$. Moreover, the unique solution from Lemma 8 can be found by Newton's method in function space, see Corollary 22.

\subsection{Results on higher regularity}

To prepare for the analysis of the optimal control problem in Section 4 we next show results on higher regularity for the solution to (10)-(12). This requires additional assumptions on the data.

\section{Assumption 11.}

- The boundary $\partial \Omega$ of $\Omega$ is of class $C^{1,1}$.

- The initial data $\varphi_{0}$ satisfies $\varphi_{0} \in C^{0,1}(\bar{\Omega})$, i.e. $\varphi_{0}$ is Lipschitz continuous.

Remark 12. In fact, the higher regularity is only required to show continuity of the trilinear form $a(\cdot, \cdot, \cdot)$ under weak convergence, see Lemma 19. We show this by using sufficient regularity of $\nabla \mu^{m}$, namely $\mu^{m} \in H^{2}$. To achieve this regularity we in turn require $C^{0,1}(\bar{\Omega})$ regularity for $\varphi^{m-1}$, appearing as a diffusion parameter in (11). Assumption 11 guarantees this regularity by guaranteeing $W^{2, p}(\Omega) \hookrightarrow C^{0,1}(\bar{\Omega})$ regularity for $\varphi^{m}$.

We start with showing Lipschitz continuity for $\varphi^{m}$ in Lemma 13 and show $H^{2}(\Omega)$-regularity for $\mu^{m}$ in Lemma 14 .

Lemma 13. Let Assumption 11 hold and let $\varphi^{m-1} \in H^{1}(\Omega) \cap C^{0,1}(\bar{\Omega})$ and $\mu^{m} \in W^{1,3}(\Omega)$ be given. Then $\varphi^{m}$, the solution to (12), satisfies $\varphi^{m} \in C^{0,1}(\bar{\Omega})$ and

$$
\left\|\varphi^{m}\right\|_{C^{0,1}(\bar{\Omega})} \leq C\left(\left\|v^{m-1}\right\|_{H^{1}(\Omega)},\left\|\varphi^{m-1}\right\|_{H^{1}(\Omega)},\left\|\varphi^{m-1}\right\|_{C^{0,1}(\bar{\Omega})}\right),
$$

where the latter is a polynomial of its arguments.

Proof. From Lemma 8 we have $\varphi^{m} \in C(\bar{\Omega}) \hookrightarrow L^{\infty}(\Omega)$. Thus we obtain $W_{+}^{\prime}\left(\varphi^{m}\right) \in L^{\infty}(\Omega)$. Further $\mu^{m} \in L^{p}(\Omega)$ for any $p<\infty$ by Sobolev embedding and $W_{-}^{\prime}\left(\varphi^{m-1}\right) \in L^{\infty}(\Omega)$ since $\varphi^{m-1} \in C^{0,1}(\bar{\Omega})$. Finally, as $\varphi^{m-1} \in C^{0,1}(\bar{\Omega})$ it holds $\left.\varphi^{m-1}\right|_{\partial \Omega} \in C^{0,1}(\partial \Omega) \hookrightarrow W^{1, \infty}(\partial \Omega) \hookrightarrow W^{1-1 / p, p}(\partial \Omega)$ for $p>n$. Combining these terms lead to $\varphi^{m} \in W^{2, p}(\Omega)$ by [31, Thm. 2.4.2.7] with $p>n$.

The stability estimate now follows from [31, Thm. 2.3.3.6] and the continuous embedding $W^{2, p}(\Omega) \hookrightarrow C^{0,1}(\bar{\Omega})$, namely

$$
\begin{aligned}
& \left\|\varphi^{m}\right\|_{C^{0,1}(\bar{\Omega})} \leq C\left\|\varphi^{m}\right\|_{W^{2, p}(\Omega)} \\
& \leq C\left(\left\|\mu^{m}+\frac{\sigma}{\epsilon} W_{+}^{\prime}\left(\varphi^{m}\right)+\frac{\sigma}{\epsilon} W_{-}^{\prime}\left(\varphi^{m-1}\right)\right\|_{L^{p}(\Omega)}+\left\|-\left(\frac{r}{\tau}+\frac{S_{\gamma}}{2}\right) \varphi^{m-1}+\gamma^{\prime}\left(\varphi^{m-1}\right)\right\|_{W^{1-\frac{1}{p}, p}(\partial \Omega)}\right) \\
& \leq C\left(\left\|\mu^{m}\right\|_{W^{1,3}(\Omega)}+\left\|\varphi^{m}\right\|_{C(\bar{\Omega})}+\left\|\varphi^{m-1}\right\|_{C(\bar{\Omega})}+\left\|\varphi^{m-1}\right\|_{C^{0,1}(\bar{\Omega})}\right) .
\end{aligned}
$$


In the last estimate we used the Lipschitz continuity of $\gamma_{u}^{\prime}$. Using Lemma 8 leads to the final result.

With the results from Lemma 13 we can now show higher regularity for $\mu^{m}$.

Lemma 14. Let Assumption 11 hold and let $\varphi^{m-1} \in H^{1}(\Omega) \cap C^{0,1}(\bar{\Omega})$ and $v^{m-1} \in H_{\sigma}(\Omega)$ be given. Then $\mu^{m} \in H^{2}(\Omega)$ and

$$
\left\|\mu^{m}\right\|_{H^{2}(\Omega)} \leq C\left(\left\|v^{m-1}\right\|_{H^{1}(\Omega)},\left\|\varphi^{m-1}\right\|_{H^{1}(\Omega)},\left\|\varphi^{m-1}\right\|_{L^{\infty}(\Omega)}\right),
$$

where the latter denotes a polynomial of its arguments.

Proof. Since $\varphi^{m-1}$ is uniformly bounded, we can cut off $\left|\varphi^{m-1}\right|^{2}$ at some some large positive value $M$ und extend it linearly without changing the actually taken values. We denote this modified function $\left|\varphi^{m-1}\right|_{M}^{2}$. Then for $\varphi^{m-1} \in C^{0,1}(\bar{\Omega})$ we have that $\left|\varphi^{m-1}\right|_{M}^{2} \in C^{0,1}(\bar{\Omega})$ is satisfied.

We have $\nabla \varphi^{m-1} v^{m-1} \in L^{2}(\Omega)$ since $\varphi^{m-1} \in H^{1}(\Omega) \hookrightarrow L^{4}(\Omega)$ and $v^{m-1} \in H_{\sigma}(\Omega) \hookrightarrow L^{4}(\Omega)^{n}$. Now the regularity result follows from [31, Thm. 2.4.2.7], while for the stability result [31, Thm. 2.3.3.6] is taken and provides

$$
\left\|\mu^{m}\right\|_{H^{2}(\Omega)} \leq C\left\|\tau^{-1}\left(\varphi^{m}-\varphi^{m-1}\right)+\nabla \varphi^{m-1} \cdot v^{m-1}\right\|_{L^{2}(\Omega)} .
$$

The final result again follows from applying Lemma 8

We can summarize Lemma 13 and Lemma 14 and state the following corollary.

Corollary 15. Let $\Omega$ and $\varphi_{0}$ satisfy Assumption 11. Then the solution to (10)-(12) satisfies for $m=1, \ldots, M$

$$
v^{m} \in H_{\sigma}(\Omega), \quad \varphi^{m} \in H^{1}(\Omega) \cap C^{0,1}(\bar{\Omega}), \quad \mu^{m} \in H^{2}(\Omega)
$$

It further holds

$$
\left\|v^{m}\right\|_{H^{1}(\Omega)}+\left\|\varphi^{m}\right\|_{H^{1}(\Omega)}+\left\|\varphi^{m}\right\|_{C^{0,1}(\bar{\Omega})}+\left\|\mu^{m}\right\|_{H^{2}(\Omega)} \leq C\left(\left\|\varphi_{0}\right\|_{H^{1}(\Omega)},\left\|\varphi_{0}\right\|_{C^{0,1}(\bar{\Omega})},\left\|v_{0}\right\|_{H^{1}(\Omega)}\right)
$$

or equivalently

$\left\|v_{\tau}\right\|_{l^{\infty}\left(H^{1}(\Omega)\right)}+\left\|\varphi_{\tau}\right\|_{l^{\infty}\left(H^{1}(\Omega)\right)}+\left\|\varphi_{\tau}\right\|_{l^{\infty}\left(C^{0,1}(\bar{\Omega})\right)}+\left\|\mu_{\tau}\right\|_{l^{\infty}\left(H^{2}(\Omega)\right)} \leq C\left(\left\|\varphi_{0}\right\|_{H^{1}(\Omega)},\left\|\varphi_{0}\right\|_{C^{0,1}(\bar{\Omega})},\left\|v_{0}\right\|_{H^{1}(\Omega)}\right)$

where the constant depends polynomially on its arguments.

Remark 16. Lemma 13 and Lemma 14 stay true, if in Assumption 11 the regularity

$$
\Omega \text { is of class } C^{1,1}
$$

is substituted by

$$
n=2 \text { and } \Omega \text { is convex and polygonally bounded. }
$$

In the latter case the reference [31, Thm. 2.4.2.7] has to be changed to [31, Thm. 4.3.2.4, Thm. 4.4.3.7]. 
Notation 17. Lemma 8 guarantees the existence of a solution operator for (10)-(12). For a further investigation, we introduce the spaces

$$
\begin{aligned}
& Y=\left(H_{\sigma}(\Omega) \times\left(H^{1}(\Omega) \cap L^{\infty}(\Omega)\right) \times W^{1,3}(\Omega)\right)^{M}, \quad y=\left(v_{\tau}, \varphi_{\tau}, \mu_{\tau}\right), \\
& Z=\left(\left(H_{\sigma}(\Omega) \times H^{1}(\Omega) \times H^{1}(\Omega)\right)^{\star}\right)^{M} .
\end{aligned}
$$

The norm of $y \in Y$ is defined by

$$
\|y\|_{Y}=\left\|\left(v_{\tau}, \varphi_{\tau}, \mu_{\tau}\right)\right\|_{Y}=\left\|v_{\tau}\right\|_{l^{\infty}\left(H^{1}(\Omega)\right)}+\left\|\varphi_{\tau}\right\|_{l^{\infty}\left(H^{1}(\Omega)\right)}+\left\|\varphi_{\tau}\right\|_{l^{\infty}\left(L^{\infty}(\Omega)\right)}+\left\|\mu_{\tau}\right\|_{l^{\infty}\left(W^{1,3}(\Omega)\right)}
$$

With the above spaces we introduce an operator $e: Y \times U \rightarrow Z$ to abbreviate (10)-(12) for $m=1, \ldots, M$ as

$$
\begin{aligned}
\langle z, e(y, u)\rangle_{Z^{\star}, Z}=\sum_{m=1}^{M} & {\left[\left(\frac{\rho^{m}+\rho^{m-1}}{2} v^{m}-\rho^{m-1} v^{m-1}, w^{m}\right)+\tau a\left(\rho^{m-1} v^{m-1}+J^{m}, v^{m}, w^{m}\right)\right.} \\
& \left.+\tau\left(2 \eta^{m} D v^{m}, D w^{m}\right)+\tau\left(\varphi^{m-1} \nabla \mu^{m}, w^{m}\right)-\tau\left(g \rho^{m}, w^{m}\right)\right] \\
& +\left[\left(\varphi^{m}-\varphi^{m-1}, \Psi^{m}\right)-\tau\left(\varphi^{m-1} v^{m-1}, \nabla \Psi^{m}\right)\right. \\
& \left.+\frac{\tau^{2}}{\rho_{\min }}\left(\left|\varphi^{m-1}\right|^{2} \nabla \mu^{m}, \nabla \Psi^{m}\right)+\tau b\left(\nabla \mu^{m}, \nabla \Psi^{m}\right)\right] \\
& +\left[\tau \sigma \epsilon\left(\nabla \varphi^{m}, \nabla \Phi^{m}\right)+\tau \frac{\sigma}{\epsilon}\left(W_{+}^{\prime}\left(\varphi^{m}\right)+W_{-}^{\prime}\left(\varphi^{m-1}\right), \Phi^{m}\right)-\tau\left(\mu^{m}, \Phi^{m}\right)\right. \\
& \left.+r\left(\varphi^{m}-\varphi^{m-1}, \Phi^{m}\right)_{\partial \Omega}+\tau\left(\frac{S_{\gamma}}{2}\left(\varphi^{m}-\varphi^{m-1}\right)+\gamma_{u}^{\prime}\left(\varphi^{m-1}\right), \Phi^{m}\right)_{\partial \Omega}\right]
\end{aligned}
$$

with $\left.z=\left(\left(w^{m}\right)_{m=1}^{M},\left(\Psi^{m}\right)_{m=1}^{M}\right),\left(\Phi^{m}\right)_{m=1}^{M}\right)$. With this notation (10)-12) for $m=1, \ldots, M$ reduces to

$$
e(y, u)=0 \in Z
$$

and Lemma 8 ensures, that for any $u \in U_{a d}^{0}$ there exists a unique $y \in Y$ such that $e(y, u)=0$ and $\|y\|_{Y} \leq C$, where $C>0$ is independent of $u \in U_{a d}^{0}$.

\subsection{Continuity under weak convergence}

Here we show that the solution operator for (10)-(12) is continuous under weak convergence and start with a preparatory lemma.

Lemma 18. Let $\left(g_{i}\right)_{i \in \mathbb{N}}$ denote a sequence that converges strongly to $g$ in $L^{p}(\Omega)$ for some $p \geq 1$. Further there exists $C>0$ such that $\left|g_{i}\right| \leq C$ independent of $i$. Let $\Psi \in L^{q}(\Omega)$ denote a fixed function with $q<\infty$.

Then $\left\|g_{i} \Psi-g \Psi\right\|_{L^{q}(\Omega)} \rightarrow 0$, i.e. $\left(g_{i} \Psi\right) \rightarrow g \Psi$ strongly in $L^{q}(\Omega)$.

Proof. This is a direct result from Lebesgue's general convergence theorem, see e.g. 10, Thm. 3.25]. Since $\left(g_{i}\right)_{i \in \mathbb{N}}$ converges strongly in some $L^{p}(\Omega)$ a subsequence converges pointwise almost everywhere, and the same holds for $\left(g_{i} \Psi\right)_{i \in \mathbb{N}}$. Further $\left|g_{i} \Psi\right|^{q} \leq C|\Psi|^{q} \in L^{1}(\Omega)$. 
From [10, Thm. 3.25] in this situation we obtain $g_{i} \Psi \in L^{q}(\Omega)$ and $\left(g_{i} \Psi\right) \rightarrow g \Psi$ strongly in $L^{q}(\Omega)$.

Lemma 19. Let Assumption 11 hold. Let $\left(u_{i}\right)_{i \in \mathbb{N}} \subset U_{\text {ad }}^{0}$ denote a weakly converging sequence to some $u \in U_{a d}^{0}$ and let $\left(y_{i}\right)_{i \in \mathbb{N}} \in Y$ denote a weakly converging subsequence to some $y \in Y$ with $e\left(y_{i}, u_{i}\right)=0$ for all $i \in \mathbb{N}$. Then $e(y, u)=0$, i.e. $e$ is continuous under weak convergence.

Proof. We discuss the operator $e$ for a fixed $m \in\{1, \ldots, M\}$ and denote subsequences by the same index $i$. All results hold for certain subsequences. We only discuss the convergence of the nonlinear terms. The linear terms directly converge by definition of weak convergence. Unless explicitly mentioned, all results are valid without the higher regularity that Assumption 11 guarantees.

\section{Convergence in equation (11):}

- It holds $\left(\varphi_{i}^{m-1} v_{i}^{m-1}, \nabla \Psi\right) \rightarrow\left(\varphi^{m-1} v^{m-1}, \nabla \Psi\right)$ by the compact embedding $H^{1}(\Omega) \hookrightarrow L^{4}(\Omega)$ that we use for $\varphi_{i}^{m-1}$ and $v_{i}^{m-1}$.

- Further, we have $\varphi_{i}^{m-1} \rightarrow \varphi^{m-1}$ strongly in $L^{2}(\Omega)$ by compact embedding $H^{1}(\Omega) \hookrightarrow L^{2}(\Omega)$ and thus pointwise almost everywhere for a subsequence. Further $\varphi_{i}^{m-1}$ is uniformly bounded in $L^{\infty}(\Omega)$. From Lemma 18 we observe $\left|\varphi_{i}^{m-1}\right|^{2} \nabla \Psi \rightarrow\left|\varphi^{m-1}\right|^{2} \nabla \Psi$ strongly in $L^{2}(\Omega)$ and thus $\left(\left|\varphi_{i}^{m-1}\right|^{2} \nabla \mu_{i}^{m}, \nabla \Psi\right) \rightarrow\left(\left|\varphi^{m-1}\right|^{2} \nabla \mu^{m}, \nabla \Psi\right)$.

\section{Convergence in equation (12):}

- The term $\left(W_{+}^{\prime}\left(\varphi_{i}^{m}\right)+W_{-}^{\prime}\left(\varphi_{i}^{m-1}\right), \Phi\right)$ converges to $\left(W_{+}^{\prime}\left(\varphi^{m}\right)+W_{-}^{\prime}\left(\varphi^{m-1}\right), \Phi\right)$ by Lebesgues's general convergence theorem, using the bounds $\left|W_{+}^{\prime}\left(\varphi^{m}\right)\right| \leq C\left(1+\left|\varphi^{m}\right|^{3}\right)$ and $\left|W_{-}^{\prime}\left(\varphi^{m-1}\right)\right| \leq$ $C\left(1+\left|\varphi^{m-1}\right|^{3}\right)$ and the compact embedding $H^{1}(\Omega) \hookrightarrow L^{5}(\Omega)$ to obtain a strongly converging subsequence.

- Further the trace operator $\operatorname{Tr}: H^{1}(\Omega) \rightarrow L^{2}(\partial \Omega)$ is compact, thus $\left.\varphi_{i}^{m}\right|_{\partial \Omega}$ is strongly converging in $L^{2}(\partial \Omega)$, see [50, Thm. 6.2].

- Using the structure of $\gamma_{u}^{\prime}\left(\varphi_{i}^{m-1}\right)=\sigma_{l g}\left(\cos \left(\theta_{e q}\right)+B_{m} u_{i}\right) \vartheta^{\prime}\left(\varphi_{i}^{m-1}\right)$ we next show that $\vartheta^{\prime}\left(\varphi_{i}^{m-1}\right) \Phi$ is strongly converging with respect to $L^{2}(\partial \Omega)$ and thus $\gamma_{u}^{\prime}\left(\varphi_{i}^{m-1}\right) \Phi \rightarrow \gamma_{u}^{\prime}\left(\varphi^{m-1}\right) \Phi$. The sequence $\vartheta^{\prime}\left(\varphi_{i}^{m-1}\right)$ converges pointwise almost everywhere and is uniformly bounded in $L^{\infty}(\partial \Omega)$ by construction. Thus by Lemma $18 \vartheta^{\prime}\left(\varphi_{i}^{m-1}\right) \Phi \rightarrow \vartheta^{\prime}\left(\varphi^{m-1}\right) \Phi$ strongly in $L^{2}(\partial \Omega)$ which yields $\left(B_{m} u_{i} \vartheta^{\prime}\left(\varphi_{i}^{m-1}\right), \Phi\right)_{\partial \Omega} \rightarrow\left(B_{m} u \vartheta^{\prime}\left(\varphi^{m-1}\right), \Phi\right)_{\partial \Omega}$, where we use that $B$ is linear and thus continuous under weak convergence.

Convergence in equation (10): Besides the trilinear form $a\left(\rho_{i}^{m-1} v_{i}^{m-1}+J_{i}^{m}, v_{i}^{m}, w\right)$ the convergence follows straightforwardly with the arguments mentioned so far.

- We first consider $\left(\left(\rho_{i}^{m-1} v_{i}^{m-1} \nabla\right) v_{i}^{m}, w\right)$. By Lemma (18) we have $\rho_{i}^{m-1} w \rightarrow \rho^{m-1} w$ strongly in $\left(L^{4}(\Omega)\right)^{n}$. Together with the compact embedding $\left(H^{1}(\Omega)\right)^{n} \hookrightarrow\left(L^{4}(\Omega)\right)^{n}$ we obtain $\left(\left(\rho_{i}^{m-1} v_{i}^{m-1} \nabla\right) v_{i}^{m}, w\right) \rightarrow$ $\left(\left(\rho^{m-1} v^{m-1} \nabla\right) v^{m}, w\right)$.

- Similarly we obtain $\left(\left(\rho_{i}^{m-1} v_{i}^{m-1} \nabla\right) w, v_{i}^{m}\right) \rightarrow\left(\left(\rho^{m-1} v^{m-1} \nabla\right) w, v^{m}\right)$ using strong convergence $\rho_{i}^{m-1} \nabla w \rightarrow \rho^{m-1} \nabla w$ in $\left(L^{2}(\Omega)\right)^{n}$. 
Finally we consider the terms including $J^{m}=-b \rho^{\prime}\left(\varphi^{m}\right) \nabla \mu^{m}$. Since $\left(y_{i}\right)$ solves (10)-(12) and due to Assumption 11 Corollary 15 applies. We stress that this is the only term, that requires the results on higher regularity.

- We start with $\left(\left(\rho^{\prime}\left(\varphi_{i}^{m}\right) \nabla \mu_{i}^{m} \nabla\right) w \cdot v_{i}^{m}\right) \rightarrow\left(\left(\rho^{\prime}\left(\varphi_{i}^{m}\right) \nabla \mu_{i}^{m} \nabla\right) w \cdot v^{m}\right)$. Here we use Lemma 18 to obtain $\rho^{\prime}\left(\varphi_{i}^{m}\right) \nabla w \rightarrow \rho^{\prime}\left(\varphi^{m}\right) \nabla w$ strongly in $\left(L^{2}(\Omega)\right)^{n}$. By compact embedding we have $v_{i}^{m} \rightarrow v^{m}$ strongly in $\left(L^{4}(\Omega)\right)^{n}$, and further from the compact embedding $H^{2}(\Omega) \hookrightarrow W^{1,4}(\Omega)$ we have strong convergence $\nabla \mu_{i}^{m} \rightarrow \nabla \mu^{m}$ in $\left(L^{4}(\Omega)\right)^{n}$. This yields the desired convergence.

- For the convergence $\left(\left(\rho^{\prime}\left(\varphi_{i}^{m}\right) \nabla \mu_{i}^{m} \nabla\right) v_{i}^{m} \cdot w\right) \rightarrow\left(\left(\rho^{\prime}\left(\varphi_{i}^{m}\right) \nabla \mu_{i}^{m} \nabla\right) v_{i}^{m} \cdot w\right)$ we first note, that $\rho^{\prime}\left(\varphi_{i}^{m}\right) w \rightarrow \rho^{\prime}\left(\varphi^{m}\right) w$ strongly in $\left(L^{6}(\Omega)\right)^{n}$ by the continuous embedding $\left(H^{1}(\Omega)\right)^{n} \hookrightarrow\left(L^{6}(\Omega)\right)^{n}$ and Lemma 18, Further we have $\nabla \mu_{i}^{m} \rightarrow \nabla \mu^{m}$ strongly in $\left(L^{3}(\Omega)\right)^{n}$ by the compact embedding $H^{2}(\Omega) \hookrightarrow W^{1,3}(\Omega)$. Together with the convergence $v_{i}^{m} \rightarrow v^{m}$ weakly in $\left(L^{2}(\Omega)\right)^{n}$ the result follows.

\subsection{Fréchet differentiability of the forward model}

By a straightforward calculation we observe that for given $u \in U_{a d}^{0}$, the operator $e(\cdot, u)$ is Gateaux differentiable with respect to the first component. The Gateaux derivative at $y=\left(\left(v^{m}\right)_{m=1}^{M},\left(\varphi^{m}\right)_{m=1}^{M},\left(\mu^{m}\right)_{m=1}^{M}\right) \in$ $Y$ in direction $d_{y}=\left(\left(d_{v}^{m}\right)_{m=1}^{M},\left(d_{\varphi}^{m}\right)_{m=1}^{M},\left(d_{\mu}^{m}\right)_{m=1}^{M}\right)$ is given by

$$
\begin{array}{r}
\left\langle e_{y}(y, u) d_{y}, z\right\rangle_{Z^{\star}, Z}=\sum_{m=1}^{M}\left[\frac{1}{2}\left(\rho^{\prime}\left(\varphi^{m}\right) d_{\varphi}^{m}, v^{m} w^{m}+v^{m+1} w^{m+1}\right)+\frac{1}{2}\left(\left(\rho^{m}+\rho^{m-1}\right) d_{v}^{m}, w^{m}\right)\right. \\
-\left(\rho^{\prime}\left(\varphi^{m}\right) d_{\varphi}^{m} v^{m}+\rho^{m} d_{v}^{m}, w^{m+1}\right) \\
+\tau a\left(\rho^{\prime}\left(\varphi^{m}\right) d_{\varphi}^{m} v^{m}+\rho^{m} d_{v}^{m}, v^{m+1}, w^{m+1}\right) \\
+\tau a\left(-b \rho^{\prime \prime}\left(\varphi^{m}\right) d_{\varphi}^{m} \nabla \mu^{m}-b \rho^{\prime}\left(\varphi^{m}\right) \nabla d_{\mu}^{m}, v^{m}, w^{m}\right) \\
+\tau a\left(\rho^{m-1} v^{m-1}+J^{m}, d_{v}^{m}, w^{m}\right) \\
+\tau\left(2 \eta^{\prime}\left(\varphi^{m}\right) d_{\varphi}^{m} D v^{m}, D w^{m}\right)+\tau\left(2 \eta^{m} D d_{v}^{m}, D w^{m}\right) \\
+\tau\left(d_{\varphi}^{m} \nabla \mu^{m+1}, w^{m+1}\right)+\tau\left(\varphi^{m-1} \nabla d_{\mu}^{m}, w^{m}\right) \\
\left.-\tau\left(g \rho^{\prime}\left(\varphi^{m}\right) d_{\varphi}^{m}, w^{m}\right)\right] \\
+\left[\left(d_{\varphi}^{m}, \Psi^{m}-\Psi^{m+1}\right)-\tau\left(d_{\varphi}^{m} v^{m}+\varphi^{m} d_{v}^{m}, \nabla \Psi^{m+1}\right)\right. \\
+\left[\tau \sigma\left(\nabla d_{\varphi}^{m}, \nabla \Phi^{m}\right)+\frac{\tau \sigma}{\epsilon}\left(W_{+}^{\prime \prime}\left(\varphi^{m}\right) d_{\varphi}^{m}, \Phi^{m}\right)+\frac{\tau \sigma}{\epsilon}\left(W_{-}^{\prime \prime}\left(\varphi^{m}\right) d_{\varphi}^{m}, \Phi^{m+1}\right)-\tau\left(d_{\mu}^{m}, \Phi^{m}\right)\right. \\
+r\left(d_{\varphi}^{m}, \Phi^{m}-\Phi^{m+1}\right) \\
\rho_{\min }+\tau\left(\frac{S_{\gamma}}{2}\left(d_{\varphi}^{m}, \Phi^{m}-\Phi^{m+1}\right) \partial \Omega\right.
\end{array}
$$

The square brackets $[\cdot]$ indicate, that the included terms stem from differentiating the same equation. Moreover, we use the convention, that functions with index $M+1$ are defined as zero. 
Theorem 20. Let $u \in U_{a d}^{0}$ be given. The operator $e(\cdot, u): Y \rightarrow Z$ is Fréchet differentiable.

Proof. As (22) describes a time stepping scheme, it is sufficient to consider only one time instance as the derivative has a lower block triangular structure. Moreover, the systems on every time instance are sequentially coupled that leads to a triangular structure also of the diagonal blocks. Therefor we start with discussing (11)-(12) and discuss (10) afterwards.

The Fréchet-differentiability of (11) - (12) without the additional terms from transport and transport decoupling directly follows as e.g. in [40]. The additional terms from the derivative with respect to $\varphi^{m-1}$ are in a lower diagonal block. Note that here it is required, that we use variations in $L^{\infty}(\Omega)$.

Since (10) is a linear system it is Fréchet differentiable.

Theorem 21. Let $u \in U_{a d}^{0}$ and $y \in Y$ be given. Then there exists a unique solution $d_{y}$ to the linear system of equations given by (23). This solution satisfies

$$
\left\|d_{y}\right\|_{Y} \leq C\left(\left\|\varphi^{0}\right\|_{H^{1}(\Omega)},\left\|\varphi^{0}\right\|_{L^{\infty}(\Omega)},\left\|v^{0}\right\|_{H^{1}(\Omega)}\right)
$$

Thus the linear operator $e_{y}(\cdot, u)$ is uniformly invertible.

Proof. Equation (23) is a time stepping scheme and on every time step we have to solve the following linear system of equations

$$
\begin{array}{r}
\left(\frac{\rho^{\prime}\left(\varphi^{m}\right) d_{\varphi}^{m}+\rho^{\prime}\left(\varphi^{m-1}\right) d_{\varphi}^{m-1}}{2} v^{m}+\frac{\rho^{m}+\rho^{m-1}}{2} d_{v}^{m}, w^{m}\right) \\
-\left(\rho^{\prime}\left(\varphi^{m-1}\right) d_{\varphi}^{m-1} v^{m-1}+\rho^{m-1} d_{v}^{m-1}, w^{m}\right) \\
+\tau a\left(\rho^{\prime}\left(\varphi^{m-1}\right) d_{\varphi}^{m-1} v^{m-1}+\rho^{m-1} d_{v}^{m-1}, v^{m}, w^{m}\right) \\
+\tau a\left(-b \rho^{\prime}\left(\varphi^{m}\right) \nabla d_{\mu}^{m}-b \rho^{\prime \prime}\left(\varphi^{m}\right) d_{\varphi}^{m} \nabla \mu^{m}, v^{m}, w^{m}\right)+\tau a\left(\rho^{m-1} v^{m-1}+J^{m}, d_{v}^{m}, w^{m}\right) \\
+\tau\left(2 \eta^{m} D d_{v}^{m}, D w^{m}\right)+\tau\left(2 \eta^{\prime}\left(\varphi^{m}\right) d_{\varphi}^{m} D v^{m}, D w^{m}\right) \\
+\tau\left(\varphi^{m-1} \nabla d_{\mu}^{m}, w^{m}\right)-\tau\left(g \rho^{\prime}\left(\varphi^{m}\right) d_{\varphi}^{m}, w^{m}\right)+\tau\left(d_{\varphi}^{m-1} \nabla \mu^{m}, w^{m}\right)=0, \\
\left(d_{\varphi}^{m}-d_{\varphi}^{m-1}, \Psi^{m}\right)-\tau\left(d_{\varphi}^{m-1} v^{m-1}+\varphi^{m-1} d_{v}^{m-1}, \nabla \Psi^{m}\right) \\
\tau \sigma \epsilon\left(\nabla d_{\varphi}^{m}, \nabla \Phi^{m}\right)+\frac{\tau \sigma}{\epsilon}\left(W_{+}^{\prime \prime}\left(\varphi^{m}\right) d_{\varphi}^{m}, \Phi^{m}\right)+\frac{\tau \sigma}{\epsilon}\left(W_{-}^{\prime \prime}\left(\varphi^{m-1}\right) d_{\varphi}^{m-1}, \Phi^{m}\right)-\tau\left(d_{\mu}^{m}, \Phi^{m}\right) \\
+r\left(d_{\varphi}^{m}-d_{\varphi}^{m-1}, \Phi^{m}\right)_{\partial \Omega}+\tau\left(\frac{S_{\gamma}}{2}\left(d_{\varphi}^{m}-d_{\varphi}^{m-1}, \Phi^{m}\right)_{\partial \Omega}+\left(\gamma_{u}^{\prime \prime}\left(\varphi^{m-1}\right) d_{\varphi}^{m-1}, \Phi^{m}\right)_{\partial \Omega}\right)=0 .
\end{array}
$$

It is sufficient to show the results for one time step only. Further we note, that (24) is decoupled from (25)-(26) and we first discuss (25)-26).

The existence for (25)-(26) follows from a Galerkin approach. Since (25)-(26) is a linear system in $d_{\varphi}^{m}, d_{\mu}^{m}$, the existence of a finite dimensional approximation follows from its uniqueness that can be shown by considering the difference between two solutions and using these differences at test functions in (25)-(26).

We next show the a-priori bound for this solution, the existence and uniqueness for (25) $-(26)$ then follows immediately by using a Galerkin approach. For given $\varphi^{m-1} \in L^{\infty}(\Omega), \varphi^{m} \in L^{\infty}(\Omega)$, 
$\mu^{m} \in W^{1,3}(\Omega), v^{m-1} \in H^{1}(\Omega), d_{\varphi}^{m-1} \in L^{\infty}(\Omega) \cap H^{1} \Omega, d_{v}^{m-1} \in H^{1}(\Omega)$ we use $\Psi^{m} \equiv d_{\mu}^{m}$ in (25) and $\Phi^{m} \equiv \frac{1}{\tau}\left(d_{\varphi}^{m}-d_{\varphi}^{m-1}\right)$ in (26) and add these equations to obtain

$$
\begin{array}{r}
\tau b\left\|\nabla d_{\mu}^{m}\right\|^{2}+\frac{\tau^{2}}{\rho_{\min }}\left(\left|\varphi^{m-1}\right|^{2} \nabla d_{\mu}^{m}, d_{\mu}^{m}\right)+\frac{\tau^{2}}{\rho_{\min }}\left(2 d_{\varphi}^{m-1} \nabla \mu^{m}, \nabla d_{\mu}^{m}\right)-\tau\left(d_{\varphi}^{m-1} v^{m-1}+\varphi^{m-1} d_{v}^{m-1}, \nabla d_{\mu}^{m}\right) \\
\sigma \epsilon\left(\nabla d_{\varphi}^{m}, \nabla\left(d_{\varphi}^{m}-d_{\varphi}^{m-1}\right)\right)+\frac{\sigma}{\epsilon}\left(W_{+}^{\prime \prime}\left(\varphi^{m}\right) d_{\varphi}^{m}, d_{\varphi}^{m}-d_{\varphi}^{m-1}\right)+\frac{\sigma}{\epsilon}\left(W_{-}^{\prime \prime}\left(\varphi^{m-1}\right) d_{\varphi}^{m-1}, d_{\varphi}^{m}-d_{\varphi}^{m-1}\right) \\
+\frac{r}{\tau}\left\|d_{\varphi}^{m}-d_{\varphi}^{m-1}\right\|_{\partial \Omega}^{2}+\frac{S_{\gamma}}{2}\left\|d_{\varphi}^{m}-d_{\varphi}^{m-1}\right\|_{\partial \Omega}^{2}+\left(\gamma_{u}^{\prime \prime}\left(\varphi^{m-1}\right) d_{\varphi}^{m-1}, d_{\varphi}^{m}-d_{\varphi}^{m-1}\right)_{\partial \Omega}=0 .
\end{array}
$$

Using $2 a(a-b)=a^{2}+(a-b)^{2}-b^{2}$, and $\left|\varphi^{m-1}\right|^{2} \geq 0$ we proceed

$$
\begin{array}{r}
\tau b\left\|\nabla d_{\mu}^{m}\right\|^{2}+\frac{\sigma \epsilon}{2}\left\|\nabla d_{\varphi}^{m}\right\|^{2}+\frac{\sigma \epsilon}{2}\left\|\nabla\left(d_{\varphi}^{m}-d_{\varphi}^{m-1}\right)\right\|^{2}-\frac{\sigma \epsilon}{2}\left\|\nabla d_{\varphi}^{m-1}\right\|^{2} \\
+\frac{\sigma}{\epsilon}\left(W_{+}^{\prime \prime}\left(\varphi^{m}\right),\left(d_{\varphi}^{m}-d_{\varphi}^{m-1}\right)^{2}\right)+\left(\frac{r}{\tau}+\frac{S_{\gamma}}{2}\right)\left\|d_{\varphi}^{m}-d_{\varphi}^{m-1}\right\|_{\partial \Omega}^{2} \\
\leq-\frac{\tau^{2}}{\rho_{\min }}\left(2 d_{\varphi}^{m-1} \nabla \mu^{m}, \nabla d_{\mu}^{m}\right)+\tau\left(d_{\varphi}^{m-1} v^{m-1}+\varphi^{m-1} d_{v}^{m-1}, \nabla d_{\mu}^{m}\right) \\
-\frac{\sigma}{\epsilon}\left(W_{+}^{\prime \prime}\left(\varphi^{m}\right) d_{\varphi}^{m-1}, d_{\varphi}^{m}-d_{\varphi}^{m-1}\right)-\frac{\sigma}{\epsilon}\left(W_{-}^{\prime \prime}\left(\varphi^{m-1}\right) d_{\varphi}^{m-1}, d_{\varphi}^{m}-d_{\varphi}^{m-1}\right)-\left(\gamma_{u}^{\prime \prime}\left(\varphi^{m-1}\right) d_{\varphi}^{m-1}, d_{\varphi}^{m}-d_{\varphi}^{m-1}\right)_{\partial \Omega} .
\end{array}
$$

From testing (25) with $\Psi \equiv 1$ we observe $\left(d_{\varphi}^{m}-d_{\varphi}^{m-1}, 1\right)=0$ and thus we enjoy mass conservation for $d_{\varphi}^{m}, m=1, \ldots, M$ and especially we can use the inequality of Poincaré-Friedrichs to estimate $\left\|d_{\varphi}^{m}-d_{\varphi}^{m-1}\right\| \leq C\left\|\nabla\left(d_{\varphi}^{m}-d_{\varphi}^{m-1}\right)\right\|$.

Using the inequalities of Hölder and Young we can thus obtain, that it holds

$$
\left\|\nabla d_{\mu}^{m}\right\|+\left\|\nabla d_{\varphi}^{m}\right\| \leq C\left(\left\|\varphi^{m}\right\|_{L^{\infty}(\Omega)},\left\|\varphi^{m-1}\right\|_{L^{\infty}(\Omega)},\left\|\nabla \mu^{m}\right\|_{L^{2}(\Omega)},\left\|v^{m-1}\right\|_{L^{2}(\Omega)},\left\|d_{\varphi}^{m-1}\right\|_{L^{\infty}(\Omega)},\left\|d_{v}^{m-1}\right\|_{L^{2}(\Omega)}\right),
$$

where $C$ is a polynomial of its arguments. Note that we only have $W_{+}^{\prime \prime}\left(\varphi^{m}\right) \geq 0$.

Using the mass conservation of $d_{\varphi}^{m}$ we in fact can bound $\left\|d_{\varphi}^{m}\right\|_{H^{1}(\Omega)}$ by the right hand side. Finally we use $\Phi^{m} \equiv 1$ in (26) together with this bound for $\left\|d_{\varphi}^{m}\right\|_{H^{1}(\Omega)}$ to obtain, that $\left(d_{\mu}^{m}, 1\right)$ is uniformly bounded from which we conclude by Poincaré-Friedrichs inequality that $\left\|d_{\mu}^{m}\right\|_{H^{1}(\Omega)}$ is also bounded and we obtain

$$
\begin{aligned}
\left\|d_{\mu}^{m}\right\|_{H^{1}(\Omega)} & +\left\|d_{\varphi}^{m}\right\|_{H^{1}(\Omega)} \\
\leq C & \left(\left\|\varphi^{m}\right\|_{L^{\infty}(\Omega)},\left\|\varphi^{m-1}\right\|_{L^{\infty}(\Omega)},\left\|\nabla \mu^{m}\right\|_{L^{2}(\Omega)},\left\|v^{m-1}\right\|_{L^{2}(\Omega)},\right. \\
& \left.\left\|d_{\varphi}^{m-1}\right\|_{L^{\infty}(\Omega)},\left\|d_{v}^{m-1}\right\|_{L^{2}(\Omega)}\right)
\end{aligned}
$$

Again by using [56, Thm. 4.8] we obtain that additionally $\left\|d_{\varphi}^{m}\right\|_{C(\bar{\Omega})}$ is bounded and the boundedness of $\left\|d_{\mu}^{m}\right\|_{W^{1,3}(\Omega)}$ follows from [22, Thm. 1.9, Thm. 5.3].

$$
\begin{gathered}
\left\|d_{\varphi}^{m}\right\|_{H^{1}(\Omega)}+\left\|d_{\varphi}^{m}\right\|_{C(\bar{\Omega})}+\left\|d_{\mu}^{m}\right\|_{W^{1,3}(\Omega)} \\
\leq C\left(\left\|\varphi^{m-1}\right\|_{L^{\infty}(\Omega)},\left\|\varphi^{m}\right\|_{L^{\infty}(\Omega)},\left\|\nabla \mu^{m}\right\|_{L^{3}(\Omega)},\left\|v^{m-1}\right\|_{L^{2}(\Omega)},\left\|v^{m}\right\|_{H^{1}(\Omega)},\right. \\
\left.\quad\left\|d_{\varphi}^{m-1}\right\|_{L^{\infty}(\Omega)},\left\|\nabla d_{\mu}^{m-1}\right\|_{L^{3}(\Omega)},\left\|d_{v}^{m-1}\right\|_{L^{2}(\Omega)}\right) .
\end{gathered}
$$


Having the unique solution to (25)-(26) at hand, in (24) we solve the same system for $d_{v}^{m}$ as we solve in (10) for $v^{m}$. The existence of a unique solution again follows immediately from Lax-Milgram's theorem, together with the bound

$$
\begin{aligned}
\left\|d_{v}^{m}\right\|_{H^{1}(\Omega)} \leq & C\left(\left\|v^{m-1}\right\|_{L^{2}(\Omega)},\left\|v^{m}\right\|_{H^{1}(\Omega)},\left\|\varphi^{m-1}\right\|_{L^{\infty}(\Omega)},\left\|\nabla \mu^{m}\right\|_{L^{3}(\Omega)},\right. \\
& \left.\left\|d_{\varphi}^{m}\right\|_{L^{\infty}(\Omega)},\left\|d_{\varphi}^{m-1}\right\|_{L^{\infty}(\Omega)},\left\|d_{v}^{m-1}\right\|_{L^{2}(\Omega)},\left\|\nabla d_{\mu}^{m}\right\|_{L^{3}(\Omega)}\right) .
\end{aligned}
$$

Iterating these estimates yields the desired result.

Corollary 22. Given $u \in U_{a d}^{0}$, Newton's method in function space can be used to find the unique solution $y \in Y$ for $e(y, u)=0$ that is guaranteed by Lemma 8 and yields local superlinear convergence.

\section{The optimization problem}

In this section we introduce and discuss the optimization problem under investigation. We assume, that we can influence the contact angle at the contact line by using a suitable control mechanism. For this we introduce a general normed and reflexive control space $U$ and an injective, linear and continuous control operator $B: U \rightarrow L^{2}\left(0, T ; L^{2}(\partial \Omega)\right)$, that maps given controls $u$ to suitable control actions, see Remark 3 . The space $U$ and the operator $B$ encapsulate the model for the actual control action.

We consider the following optimal control problem

$$
\begin{gathered}
\min _{u \in U, y \in Y} J(y, u)=\frac{1}{2} \int_{0}^{T} \int_{\Omega}\left|\varphi_{\tau}-\varphi_{d}\right|^{2} \mathrm{dx} \mathrm{dt}+\frac{\alpha}{2}\|B u\|_{L^{2}\left(0, T ; L^{2}(\partial \Omega)\right)}^{2} \\
\text { subject to } e(y, u)=0, \\
\cos \left(\theta_{\min }\right) \leq B u+\cos \left(\theta_{e q}\right) \leq \cos \left(\theta_{\max }\right) .
\end{gathered}
$$

for a constant $\alpha>0$, where $\varphi_{d}$ denotes a given distribution of the phases, that we want to obtain. Here $0<\theta_{\min }<\theta_{\max }<\pi$ are given minimum and maximum static contact angles that can be achieved and we define

$$
U_{a d}:=\left\{u \in U \mid \cos \left(\theta_{\min }\right) \leq B u+\cos \left(\theta_{e q}\right) \leq \cos \left(\theta_{\max }\right)\right\} \subset U_{a d}^{0} .
$$

Since $B$ is injective, $U_{a d}$ is a bounded set. Here we use $\|B u\|_{L^{2}\left(0, T ; L^{2}(\partial \Omega)\right)}^{2}$ as regularization in $(\mathbb{P})$, because its understanding as deviation from the equilibrium angle makes it easier to interprete than the common regularization $\|u\|_{U}^{2}$. Finally we note, that $\varphi_{\tau}$ is piecewise constant with repect to time and thus it holds

$$
\frac{1}{2} \int_{0}^{T} \int_{\Omega}\left|\varphi_{\tau}-\varphi_{d}\right|^{2} \mathrm{dxdt}=\frac{1}{2} \sum_{m=1}^{M} \tau \int_{\Omega}\left|\varphi^{m}-\varphi_{d}^{m}\right|^{2} \mathrm{dx}
$$

where $\varphi_{d}^{m}:=\frac{1}{\tau} \int_{t_{m-1}}^{t_{m}} \varphi_{d}(t) \mathrm{dt}$.

We can now use the results from Section 3.2 and Section 3.3 to show the existence of at least one optimal control to $(\mathbb{P})$ and to state first order optimality conditions. 
Theorem 23 (Existence of an optimal solution). There exists at least one optimal solution $\left(u^{\star}, y^{\star}\right)$ to $(\mathrm{P})$.

Proof. This follows from the direct method by considering an infimizing sequence and using Lemma 19 . We refer to [43, Ass. 1.44, Thm. 1.45].

Theorem 24 (First order optimality conditions). Let $(\bar{y}, \bar{u})$ denote a solution to $(\mathbb{P})$. Then it satisfies the following first order optimality conditions with some adjoint variable $\bar{p} \in Z^{\star}$.

$$
\begin{aligned}
e(\bar{y}, \bar{u}) & =0 \\
e_{y}(\bar{y}, \bar{u})^{\star} \bar{p} & =-\left(\bar{\varphi}_{\tau}-\varphi_{d}\right) \\
\bar{u} \in U_{a d}, \quad\left\langle\alpha B^{\star} B u(\bar{u})+e_{u}(\bar{y}, \bar{u})^{\star} \bar{p}, u-\bar{u}\right\rangle_{U^{\star}, U} & \geq 0 \quad \forall u \in U_{a d} .
\end{aligned}
$$

Here $e_{y}(\bar{y}, \bar{u})^{\star}$ denotes the adjoint operator of $e_{y}(\bar{y}, \bar{u})$ and correspondingly for $e_{u}(\bar{y}, \bar{u})^{\star}$.

Proof. See [43, Cor. 1.3].

For a practical implementation, we state the optimality system from Theorem 24 explicitly in our situation and start with the adjoint equation $e_{y}(\bar{y}, \bar{u})^{\star} \bar{p}=-\left(\bar{\varphi}_{\tau}-\varphi_{d}\right)$. This equation abbreviates a time stepping scheme, which steps backwards in time. On every time instance the adjoint Navier-Stokes and the adjoint Cahn-Hilliard equation are sequentially coupled, where the adjoint Navier-Stokes equation can be solved independently of the adjoint Cahn-Hilliard equation. As the adjoint equation encodes a time stepping scheme, we just state one step and note the convention, that terms with index $M+1$ are defined as zero. We denote the adjoint velocity field by $p_{v}$, the adjoint phase field by $p_{\varphi}$ and the adjoint chemical potential as $p_{\mu}$. The test functions are again denoted by $w, \Psi$, and $\Phi$. On time instance $m=M, \ldots, 1$ the adjoint system is given by 
the following time stepping scheme, backwards in time

$$
\begin{gathered}
\left(\frac{\rho^{m}+\rho^{m-1}}{2} p_{v}^{m}-\rho^{m} p_{v}^{m+1}, w^{m}\right) \\
+\tau a\left(\rho^{m} w^{m}, v^{m+1}, p_{v}^{m+1}\right)+\tau a\left(\rho^{m-1} v^{m-1}+J^{m}, w^{m}, p_{v}^{m}\right) \\
+\tau\left(2 \eta^{m} D w^{m}, D p_{v}^{m}\right)-\tau\left(\varphi^{m} w^{m}, \nabla p_{\mu}^{m+1}\right)=0, \\
\tau a\left(-b \rho^{\prime}\left(\varphi^{m}\right) \nabla \Psi^{m}, v^{m}, p_{v}^{m}\right)+\tau\left(\varphi^{m-1} \nabla \Psi^{m}, p_{v}^{m}\right) \\
+\frac{\tau^{2}}{\rho_{\min }}\left(\left|\varphi^{m-1}\right|^{2} \nabla \Psi^{m}, \nabla p_{\mu}^{m}\right)+\tau b\left(\nabla \Psi^{m}, \nabla p_{\mu}^{m}\right)-\tau\left(\Psi^{m}, p_{\varphi}^{m}\right)=0, \\
\frac{1}{2}\left(\rho^{\prime}\left(\varphi^{m}\right) \Phi^{m}, v^{m} p_{v}^{m}+v^{m+1} p_{v}^{m+1}\right)-\left(\rho^{\prime}\left(\varphi^{m}\right) \Phi^{m} v^{m}, p_{v}^{m+1}\right) \\
+\tau a\left(-b \rho^{\prime \prime}\left(\varphi^{m}\right) \Phi^{m} \nabla \mu^{m}, v^{m}, p_{v}^{m}\right)+\tau a\left(\rho^{\prime}\left(\varphi^{m}\right) \Phi^{m} v^{m}, v^{m+1}, p_{v}^{m+1}\right) \\
+\tau\left(2 \eta^{\prime}\left(\varphi^{m}\right) \Phi^{m} D v^{m}, D p_{v}^{m}\right) \\
+\tau\left(\Phi^{m} \nabla \mu^{m+1}, p_{v}^{m+1}\right)-\tau\left(g \rho^{\prime}\left(\varphi^{m}\right) \Phi^{m}, p_{v}^{m}\right) \\
+\left(\Phi^{m}, p_{\mu}^{m}-p_{\mu}^{m+1}\right)-\tau\left(\Phi^{m} v^{m}, \nabla p_{\mu}^{m+1}\right)+\frac{\tau^{2}}{\rho_{\min }}\left(2 \Phi^{m} \nabla \mu^{m+1}, \nabla p_{\mu}^{m+1}\right) \\
+\tau \sigma \epsilon\left(\nabla \Phi^{m}, \nabla p_{\varphi}^{m}\right)+\frac{\tau \sigma}{\epsilon}\left(W_{+}^{\prime \prime}\left(\varphi^{m}\right) \Phi^{m}, p_{\varphi}^{m}\right)+\frac{\tau \sigma}{\epsilon}\left(W_{-}^{\prime \prime}\left(\varphi^{m}\right) \Phi^{m}, p_{\varphi}^{m+1}\right) \\
+r\left(\Phi^{m}, p_{\varphi}^{m}-p_{\varphi}^{m+1}\right) \partial \Omega \\
+\tau\left(\frac{S_{\gamma}}{2}\left(\Phi^{m}, p_{\varphi}^{m}-p_{\varphi}^{m+1}\right) \partial \Omega+\left(\gamma_{u}^{\prime \prime}\left(\varphi^{m}\right) \Phi^{m}, p_{\varphi}^{m+1}\right) \partial \Omega\right)=-\tau\left(\varphi^{m}-\varphi_{d}^{m}, \Phi^{m}\right),
\end{gathered}
$$

where $\varphi_{d}^{m}:=\frac{1}{\tau} \int_{t_{m-1}}^{t_{m}} \varphi_{d}(t) \mathrm{dt}$.

For the optimality condition $\left\langle\alpha B^{\star} B(\bar{u})+e_{u}(\bar{y}, \bar{u})^{\star} \bar{p}, u-\bar{u}\right\rangle_{U^{\star}, U} \geq 0 \quad \forall u \in U_{a d}$ we obtain the following equation

$$
\begin{aligned}
& \alpha\left\langle B^{\star} B \bar{u}, u-\bar{u}\right\rangle_{U^{\star}, U}+\sum_{m=1}^{M}\left\langle B_{m}^{\star}\left(\tau \sigma_{l g} \vartheta^{\prime}\left(\varphi^{m-1}\right) p_{\varphi}^{m}\right), u-\bar{u}\right\rangle_{U^{\star}, U} \\
= & \alpha\left\langle B^{\star} B \bar{u}, u-\bar{u}\right\rangle_{U^{\star}, U}+\left\langle B^{\star}\left(\sum_{m=1}^{M} \sigma_{l g} \vartheta^{\prime}\left(\varphi^{m-1}\right) p_{\varphi}^{m} \chi_{m}\right), u-\bar{u}\right\rangle_{U^{\star}, U} \geq 0,
\end{aligned}
$$

where $\chi_{m}$ denotes the characteristic function of the interval $\left(t_{m-1}, t_{m}\right)$.

\section{Numerical Experiment: How to Make Water Run Uphill}

In this section we demonstrate the general applicability of our approach and framework. As a test example, we consider the setup illustrated in Figure 1 in the introduction. As observed in very famous experiments by Chaudhury and Whitesides [18], it is possible to push the droplet uphill against gravity by choosing a specific contact angle distribution between solid, droplet and surrounding fluid. In addition, this example is inspired by the work reported in [6], where the heat transfer into a sliding and pinned droplet is characterised, and [55], where drops are trapped due to steep changes in the contact angle. The pinning of a sliding droplet at a specific position on a solid 
surface while maintaining a desired shape has interesting implications for technical applications. At the same time, this is a challenging task. Note, that in practical applications, the control patches can represent electrodes. Again we refer to [49] for details on the technical implementation.

\subsubsection{Implementation}

For the spatial discretization of (10)-(12), we consider the standard finite element concept and start with considering a subdivision of $\Omega$ into triangles. On this triangulation we use piecewise linear and globally continuous functions for $\varphi$ and $\mu$. To deal with the constraint of solenoidal velocity fields, we add the pressure $p$ as an additional variable. To discretize pressure and velocity, we use piecewise linear and globally continuous functions for $p$ respectively piecewise quadratic and globally continuous functions for $v$ (i.e., we use Taylor-Hood elements for the solution of the NavierStokes part). We refer to [17] for more information on the implementation. The finite elements are provided by the toolbox FEniCS 2019.1.0 [9, 48]. For the solution of the arising nonlinear and linear systems and subsystems the software suite PETSc 3.8.4 [14 16] together with the direct linear solver MUMPS 5.1.1 [11, 12] are utilized. Note, that we do not apply any preconditioning or subiterations except for the Newton iterations, appearing from the nonlinearity $W_{+}\left(\varphi^{m}\right)$. To solve the optimization problem, we use IPOPT 3.12 [57, 58] with options set to default values.

\subsection{Setup}

A single droplet (solid line in Figure 1) is placed on an inclined surface. If no control action is taken, the droplet slides down the surface driven by gravity. Using given contact angle distributions, we control the advancing and receding contact angles $\theta_{1}$ and $\theta_{2}$ while the droplet slides along the surface. In this way, we impose the desired shape and position of the droplet at any time (dashed line) over a time horizon $I=(0, T)$ with $T=5$.

We model this setup as follows. In a rectangular domain $\Omega=(0,1) \times(0,0.5)$, a liquid, capshaped droplet $\varphi_{0}$ with radius $r_{0}=0.25$ is placed at $m=(0.375,0)$ on a smooth, solid surface (represented by the boundary $(0,1) \times\{0\}$ ) with an equilibrium contact angle of $\theta_{e q}=90^{\circ}$. The explicit definition of $\varphi_{0}$ is stated below. The inclination angle of the plate to the horizontal is $\alpha=-15^{\circ}$, which is modelled by a corresponding inclination angle of the gravitational force.

We have no-slip boundary conditions for the velocity on the left and right side and free-slip on the top side. The condition (6) is applied on the bottom boundary, i.e. $(0,1) \times\{0\}$.

Table 1 lists the applied parameter values (the values are taken from [17, 45]) for the physical and numerical parameters in model (17)-(7). The density of the droplet is greater than the density of the surrounding fluid and since the inclination is negative gravity pulls the droplet down.

\begin{tabular}{cccccccc|cc}
$\sigma_{l g}$ & $\rho_{l}$ & $\rho_{g}$ & $\eta_{l}$ & $\eta_{g}$ & $g$ & $\alpha$ & $r$ & $\epsilon$ & $b$ \\
\hline 24.5 & 1000 & 100 & 10 & 1 & 0.98 & $-0.15^{\circ}$ & 0.35 & $2 \times 10^{-2}$ & $2 \times 10^{-5}$
\end{tabular}

Table 1: Physical and numerical parameters of droplet, surrounding fluid and solid surface.

Note, that $\sigma_{l g}$ denotes the physical surface tension that has to be scaled by the constant $c_{W}$ in (41) that depends on the free energy potential $W$. Here we use

$$
W(\varphi):= \begin{cases}\frac{1}{4}\left(1-\varphi^{2}\right)^{2} & \text { if }|\varphi| \leq 1 \\ (|\varphi|-1)^{2} & \text { if }|\varphi|>1\end{cases}
$$


together with the convex-concave splitting

$$
W_{+}(\varphi):=\left\{\begin{array}{ll}
\frac{1}{4} \varphi^{4}-\frac{1}{4} & \text { if }|\varphi| \leq 1, \\
\frac{1}{2}\left(3 \varphi^{2}-4|\varphi|+1\right) & \text { if }|\varphi|>1,
\end{array} \quad W_{-}(\varphi):=\frac{1}{2}\left(1-\varphi^{2}\right)\right.
$$

This choice leads to $c_{W}=\frac{3}{2 \sqrt{2}}$ and $\Phi_{0}(z)=\tanh (z / \sqrt{2})$, see Remark 2 and [17, Rem. 2]. Using $\Phi_{0}$ we define $\varphi_{0}(x):=\Phi_{0}\left(\left(\|x-m\|-r_{0}\right) / \epsilon\right)$.

Exemplarily, we use the dashed line in Figure 3 as the desired and constant shape $\varphi_{d}$. It is created by placing a liquid, cap-shaped droplet with radius $r_{d}=0.25$ at $m_{d}=(0.625,0)$ and initial contact angle of $90^{\circ}$ on a perfectly horizontal surface (no inclination with $\alpha=0^{\circ}$ ). Thus we have $\varphi_{d}(x)=\Phi_{0}\left(\left(\left\|x-m_{d}\right\|-r_{d}\right) / \epsilon\right)$. Then the static contact angle is set to $\theta_{e q}=135^{\circ}$ and the forward model is simulated until the droplet reaches its equilibrium shape. In absence of any inclination the droplet does not slide along the surface. Note, that setting $\theta_{e q}=135^{\circ}$ is equivalent to simulating with the constant control $B u=B u+\cos \left(90^{\circ}\right)=\cos \left(135^{\circ}\right)$. The bounds for the optimizer are set to $\cos \left(\theta_{\min }\right)=-0.9$ and $\cos \left(\theta_{\max }\right)=0.9$.

\subsection{The control space $U$}

We model the control by a linear combination of fixed controls. Subsequently, we optimize the coefficients in this linear combination, compare [29]. These fixed controls either depend on time or on space. We note, that we can describe controls that only depend on time, controls that only depend on space and controls that depend on both space and time by such a model.

Let $g_{r} \in L^{2}(0, T), r=1, \ldots, R$, denote $R$ given time depending functions, and let $f_{s} \in L^{2}(\partial \Omega)$, $s=1, \ldots, S$, denote $S$ given spatially depending functions. We define $U=\mathbb{R}^{R S}$ and $B: U \rightarrow$ $L^{2}\left(0, T ; L^{2}(\partial \Omega)\right)$ by

$$
B u=\sum_{r=1}^{R} \sum_{s=1}^{S} u_{r s} g_{r}(t) f_{s}(x)
$$

where $u_{r s}$ denote the entries of $u \in \mathbb{R}^{R S}$ in a suitable ordering. The adjoint operator $B^{\star}$ : $L^{2}\left(0, T ; L^{2}(\partial \Omega)\right) \rightarrow U$ is for an arbitrary $p \in L^{2}\left(0, T ; L^{2}(\partial \Omega)\right)$ given by

$$
\left(B^{\star} p\right)_{r s}=\left(p, g_{r}(t) f_{s}(x)\right)_{L^{2}\left(0, T ; L^{2}(\partial \Omega)\right)}
$$

Note that we can set $R=1$ and $g_{1}(t)=1$ to model controls, that only depend on space and $S=1$ and $f_{s}(x)=1$ to model controls, that only depend on time.

In the following we use $S=10$, i.e., ten equally sized control patches, and $R=5$ to allow the control to switch at five equally distributed instances in time. Thus we use $f_{s}(x)=\chi_{\left(\frac{1}{10} s, \frac{1}{10}(s+1)\right)}(x)$, $s=0, \ldots, 9$ and $g_{r}(t)=\chi_{(r, r+1)}(t), r=0, \ldots, 4$, where $\chi_{J}$ denotes the characteristic function of the interval $J$.

\subsection{Results}

In Figure 3 we show numerical results that indicate that our approach is successful in the present situation. We present phase fields by their zero-level isoline. At first we demonstrate what happens to the droplet on the inclined surface without any control at all ( $B u=0$ for all times), see the 
first column in Figure 3. Secondly, one might naively set the control to the finally desired contact angle of $135^{\circ}$. This is displayed in the second column. As expected in both cases the droplet starts running downhill. Subsequently, the droplets are far from the desired shape as well as the desired position at the specified time. Certainly, finding the right control actions by trial-and-error is cumbersome. Furthermore, we are not interested in gaining any control but one control that is optimal with respect to the cost functional.

Finally, in the third column of Figure 3 the droplet's evolution is shown, if an optimal control is applied. We notice, that the droplet runs uphill and it matches the desired shape at the desired position very well for $t=5$. Comparing this to the naive approaches with $B u=0$ and $B u=-0.7071$ the improvement is tremendous. During the sliding and pinning the droplet exhibits multiple unusual shapes due to the strong impact of the control actions on the droplet. The control actions $B u$ in each time interval are shown in the last column of Figure 3 It is striking how complex these optimal controls $B u$ are. While pulled by gravity, the droplet's contact points are forced to recede and spread multiple times. See for example the stripe between 0.8 to 0.9 at the time intervals 3 to 4 and 4 to 5 . The control action jumps from large positive values (spreading, so the droplet moves uphill) to negative values (receding, so the droplet meets the desired shape).

\section{Conclusion}

In this work we considered an optimal control problem for the shape and position of droplets sliding on solid surfaces. Based on our studies in [17] on numerical schemes for two-phase flows involving moving contact line dynamics, we chose a detailed phase field model as the physical constraint. We showed higher regularity for the unique solution to this time discretization scheme for this highly nonlinear system and were able to proof existence of solutions to the corresponding optimal control problem. Further we derived first order optimality conditions that we used in the quasi-Newton algorithm of the interior-point solver IPOPT.

To demonstrate our approach we considered the active control of a sliding droplet using temporally and spatially varying contact angle distributions. In this basic example common for dropletbased microfluidics the droplet slides on an inclined surface uphill against gravity. The final droplet matched the desired shape and position almost perfectly. To our knowledge the simultaneous control of the optimal shape and position of sliding droplets on solid surfaces has not been previously demonstrated. Our work indicates that the active control of contact angles is a powerful approach towards controlled transport of droplets in microfluidic applications. In future work, we investigate the particular optimization problem and the whole control process and incorporate additional constraints that might stem from manufacturing or practical application.

\section{Acknowledgments}

We thank Constantin Christof and Johannes Pfefferer for very fruitful discussions on the regularity theory and convergence theory used in Section 3 .

\section{References}

[1] Abels, H. and Breit, D. (2016). Weak Solutions for a Non-Newtonian Diffuse Interface Model with Different Densities. Nonlinearity, 29:3426-3453. 

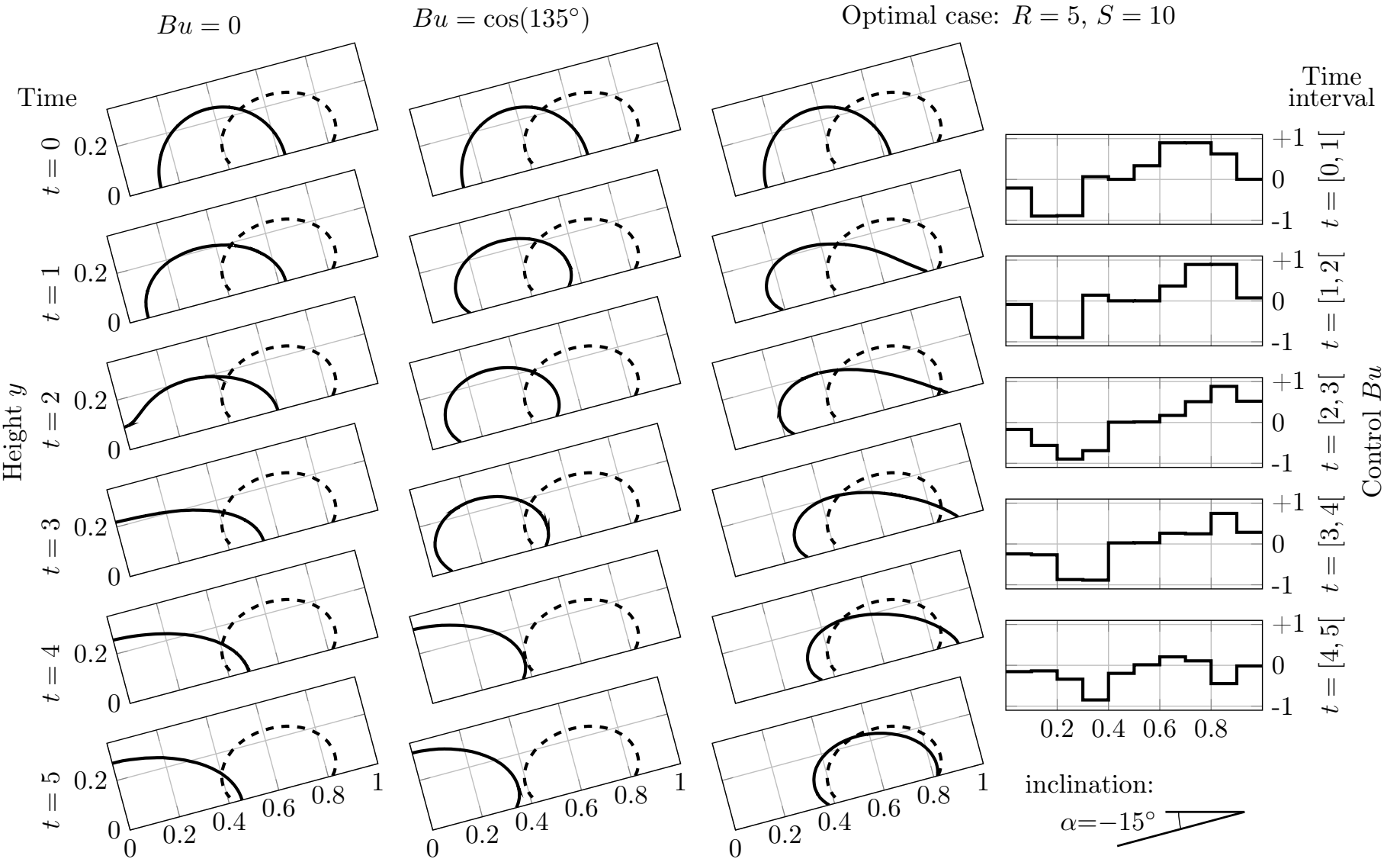

Position on plate $x$

Figure 3: Development of the sliding and pinned droplets over time on a plate with an inclination to the horizontal of $\alpha=-15^{\circ}$ : no control action with $B u=0$ (first column), constant control action with $B u=\cos \left(135^{\circ}\right)$ (second column) and optimal control action for $R=5$ and $S=10$ (third column). In the fourth column the optimal control action is displayed for the time intervals. The desired shape and position of the droplet $\varphi_{d}$ is included as its zero level set as dashed line. 
[2] Abels, H., Depner, D., and Garcke, H. (2013a). Existence of weak solutions for a diffuse interface model for two-phase flows of incompressible fluids with different densities. Journal of Mathematical Fluid Mechanics, 15(3):453-480.

[3] Abels, H., Depner, D., and Garcke, H. (2013b). On an incompressible Navier-Stokes / CahnHilliard system with degenerate mobility. Annales de l'Institut Henri Poincaré (C) Non Linear Analysis, 30(6):1175-1190.

[4] Abels, H., Garcke, H., and Grün, G. (2012). Thermodynamically consistent, frame indifferent diffuse interface models for incompressible two-phase flows with different densities. Mathematical Models and Methods in Applied Sciences, 22(3):1150013(40).

[5] Adams, R. A. and Fournier, J. H. F. (2003). Sobolev Spaces, second edition, volume 140 of Pure and Applied Mathematics. Elsevier.

[6] Al-Sharafi, A., Yilbas, B. S., Ali, H., and Alaqeeli, N. (2018). A Water Droplet Pinning and Heat Transfer Characteristics on an Inclined Hydrophobic Surface. Scientific Reports, 8(1):3061.

[7] Aland, S. (2014). Time integration for diffuse interface models for two-phase flow. Journal of Computational Physics, 262:58-71.

[8] Aland, S. and Chen, F. (2016). An efficient and energy stable scheme for a phase-field model for the moving contact line problem. International Journal for Numerical Methods in Fluids, 81:657-671.

[9] Alnæs, M., Blechta, J., Hake, J., Johansson, A., Kehlet, B., Logg, A., Richardson, C., Ring, J., Rognes, M., and Wells, G. (2015). The fenics project version 1.5. Archive of Numerical Software, $3(100)$.

[10] Alt, H. (2016). Linear Functional Analysis. Springer.

[11] Amestoy, P. R., Duff, I. S., Koster, J., and L'Excellent, J.-Y. (2001). A fully asynchronous multifrontal solver using distributed dynamic scheduling. SIAM Journal on Matrix Analysis and Applications, 23(1):15-41.

[12] Amestoy, P. R., Guermouche, A., L'Excellent, J.-Y., and Pralet, S. (2006). Hybrid scheduling for the parallel solution of linear systems. Parallel Computing, 32(2):136-156.

[13] Antil, H., Hintermüller, M., Nochetto, R., Surowiec, T., and Wegner, D. (2017). Finite horizon model predictive control of electrowetting on dielectric with pinning. Interfaces and Free Boundaries, 19(1):1-30.

[14] Balay, S., Abhyankar, S., Adams, M. F., Brown, J., Brune, P., Buschelman, K., Dalcin, L., Eijkhout, V., Gropp, W. D., Kaushik, D., Knepley, M. G., May, D. A., McInnes, L. C., Mills, R. T., Munson, T., Rupp, K., Sanan, P., Smith, B. F., Zampini, S., Zhang, H., and Zhang, H. (2018a). PETSc Web page. http://www.mcs.anl.gov/petsc

[15] Balay, S., Abhyankar, S., Adams, M. F., Brown, J., Brune, P., Buschelman, K., Dalcin, L., Eijkhout, V., Gropp, W. D., Kaushik, D., Knepley, M. G., May, D. A., McInnes, L. C., Mills, R. T., Munson, T., Rupp, K., Sanan, P., Smith, B. F., Zampini, S., Zhang, H., and Zhang, H. (2018b). PETSc users manual. Technical Report ANL-95/11 - Revision 3.9, Argonne National Laboratory. 
[16] Balay, S., Gropp, W. D., McInnes, L. C., and Smith, B. F. (1997). Efficient management of parallelism in object oriented numerical software libraries. In Arge, E., Bruaset, A. M., and Langtangen, H. P., editors, Modern Software Tools in Scientific Computing, pages 163-202. Birkhäuser Press.

[17] Bonart, H., Kahle, C., and Repke, J.-U. (2019). Comparison of energy stable simulation of moving contact line problems using a thermodynamically consistent Cahn-Hilliard Navier-Stokes model. Journal of Computational Physics, 399:108959.

[18] Chaudhury, M. K. and Whitesides, G. M. (1992). How to Make Water Run Uphill. Science, 256(5063):1539-1541.

[19] Cheng, X., Promislow, K., and Wetton, B. (2019). Asymptotic Behaviour of Time Stepping Methods for Phase Field Models. arxiv: 1905.02299v1.

[20] Colli, P., Gilardi, G., and Sprekels, J. (2017). On a Cahn-Hilliard system with convection and dynamic boundary conditions. Annali di Matematica Pura ed Applicata, pages 1-31.

[21] Colli, P. and Signori, A. (2019). Boundary control problem and optimality conditions for the Cahn-Hilliard equation with dynamic boundary conditions. arXiv: 1905.00203v1.

[22] Dhamo, V. (2012). Optimal boundary control of quasilinear elliptic partial differential equations: theory and numerical analysis.

[23] Epstein, A. K., Hochbaum, A. I., Kim, P., and Aizenberg, J. (2011). Control of bacterial biofilm growth on surfaces by nanostructural mechanics and geometry. Nanotechnology, 22(49):494007.

[24] Frigeri, S., Grasselli, M., and Sprekels, J. (2018). Optimal distributed control of twodimensional nonlocal Cahn-Hilliard-Navier-Stokes systems with degenerate mobility and singular potential. Applied Mathematics and Optimization.

[25] Fumagalli, I., Parolini, N., and Verani, M. (2017). Optimal control in ink-jet printing via instantaneous control. Computers and Fluids, 172:264-273.

[26] Gal, C. G., Grasselli, M., and Miranville, A. (2016). Cahn-Hilliard-Navier-Stokes systems with moving contact lines. Calculus of Variations and Partial Differential Equations, 55(3):50.

[27] Gao, M. and Wang, X.-P. (2012). A gradient stable scheme for a phase field model for the moving contact line problem. Journal of Computational Physics, 231(4):1372 - 1386.

[28] Garcke, H., Hinze, M., and Kahle, C. (2016). A stable and linear time discretization for a thermodynamically consistent model for two-phase incompressible flow. Applied Numerical Mathematics, 99:151-171.

[29] Garcke, H., Hinze, M., and Kahle, C. (2019). Optimal control of time-discrete two-phase flow driven by a diffuse-interface model. ESAIM: Control, Optimisation and Calculus of Variations, $25: 13$.

[30] Gräßle, C., Hintermüller, M., Hinze, M., and Keil, T. (2019). Simulation and Control of a Nonsmooth Cahn-Hilliard Navier-Stokes System. arXiv e-prints, page arXiv:1907.04285. 
[31] Grisvard, P. (2011). Elliptic Problems in Nonsmooth Domains. Classics in Applied Mathematics. SIAM.

[32] Grün, G. (2013). On convergent schemes for diffuse interface models for two-phase flow of incompressible fluids with general mass densities. SIAM Journal on Numerical Analysis, 51(6):3036-3061.

[33] Grün, G., Guillén-Gonzáles, F., and Metzger, S. (2016). On Fully Decoupled Convergent Schemes for Diffuse Interface Models for Two-Phase Flow with General Mass Densities. Communications in Computational Physics, 19(5):1473-1502.

[34] Grün, G. and Klingbeil, F. (2014). Two-phase flow with mass density contrast: Stable schemes for a thermodynamic consistent and frame indifferent diffuse interface model. Journal of Computational Physics, 257(A):708-725.

[35] Guillén-Gonzáles, F. and Tierra, G. (2014). Splitting schemes for a Navier-Stokes-CahnHilliard model for two fluids with different densities. Journal of Computational Mathematics, $32(6): 643-664$.

[36] Guillén-González, F. and Tierra, G. (2013). On linear schemes for a Cahn-Hilliard diffuse interface model. Journal of Computational Physics, 234:140-171.

[37] He, Q., Glowinski, R., and Wang, X.-P. (2011). A least-squares/finite element method for the numerical solution of the Navier-Stokes-Cahn-Hilliard system modeling the motion of the contact line. Journal of Computational Physics, 230(12):4991-5009.

[38] Hintermüller, M., Hinze, M., and Kahle, C. (2013). An adaptive finite element MoreauYosida-based solver for a coupled Cahn-Hilliard/Navier-Stokes system. Journal of Computational Physics, 235:810-827.

[39] Hintermüller, M., Hinze, M., Kahle, C., and Keil, T. (2018). A goal-oriented dual-weighted adaptive finite element approach for the optimal control of a nonsmooth Cahn-Hilliard-NavierStokes system. Optimization and Engineering, 19(3):629-662.

[40] Hintermüller, M., Hinze, M., and Tber, M. H. (2011). An adaptive finite element MoreauYosida-based solver for a non-smooth Cahn-Hilliard problem. Optimization Methods and Software, 25(4-5):777-811.

[41] Hintermüller, M. and Keil, T. (2019). Optimal control of geometric partial differential equations. WIAS preprint-2612.

[42] Hintermüller, M., Keil, T., and Wegner, D. (2017). Optimal Control of a Semidiscrete CahnHilliard-Navier-Stokes System with Nonmatched Fluid Densities. SIAM Journal on Control and Optimization, 55(3):1954-1989.

[43] Hinze, M., Pinnau, R., Ulbrich, M., and Ulbrich, S. (2009). Optimization with PDE constraints, volume 23 of Mathematical Modelling: Theory and Applications. Springer.

[44] Hou, L., Smith, N. R., and Heikenfeld, J. (2007). Electrowetting manipulation of any optical film. Applied Physics Letters, 90(25):251114. 
[45] Hysing, S., Turek, S., Kuzmin, D., Parolini, N., Burman, E., Ganesan, S., and Tobiska, L. (2009). Quantitative benchmark computations of two-dimensional bubble dynamics. International Journal for Numerical Methods in Fluids, 60(11):1259-1288.

[46] Knopf, P. and Ebenbeck, M. (2019). Optimal control theory and advanced optimality conditions for a diffuse interface model of tumor growth. ESAIM: Control, Optimisation and Calculus of Variations.

[47] Laurain, A. and Walker, S. W. (2015). Droplet Footprint Control. SIAM Journal on Control and Optimization, 53(2):771-799.

[48] Logg, A., Mardal, K.-A., and Wells, G., editors (2012). Automated Solution of Differential Equations by the Finite Element Method - The FEniCS Book, volume 84 of Lecture Notes in Computational Science and Engineering. Springer.

[49] Mugele, F. and Heikenfeld, J. (2018). Electrowetting. Wiley-VCH Verlag GmbH \& Co. KGaA, Weinheim, Germany.

[50] Nečas, J. (2012). Direct Methods in the Theory of Elliptic Equations. Springer.

[51] Pollack, M. G., Shenderov, A. D., and Fair, R. B. (2002). Electrowetting-based actuation of droplets for integrated microfluidics. Lab on a Chip, 2(2):96.

[52] Qian, T., Wang, X.-P., and Sheng, P. (2006). A variational approach to moving contact line hydrodynamics. Journal of Fluid Mechanics, 564:333-360.

[53] Shen, J., Xu, J., and Yang, J. (2018). The scalar auxiliary variable (SAV) approach for gradient flows. Journal of Computational Physics, 353:407 - 416.

[54] Shen, J., Yang, X., and Yu, H. (2015). Efficient energy stable numerical schemes for a phase field moving contact line model. Journal of Computational Physics, 284:617-630.

[55] 't Mannetje, D., Ghosh, S., Lagraauw, R., Otten, S., Pit, A., Berendsen, C., Zeegers, J., van den Ende, D., and Mugele, F. (2014). Trapping of drops by wetting defects. Nature Communications, $5(1): 3559$.

[56] Tröltzsch, F. (2005). Optimale Steuerung partieller Differentialgleichungen. Vieweg Verlag, Wiesbaden.

[57] Wächter, A. and Biegler, L. T. (2006). On the implementation of an interior-point filter linesearch algorithm for large-scale nonlinear programming. Mathematical Programming, 106(1):2557.

[58] Waechter, A., Laird, C., and Contributors (2019). IPOPT webpage. https://github.com/coin-or/Ipopt.

[59] Wu, X., van Zwieten, G., and van der Zee, K. (2014). Stabilized second-order convex splitting schemes for Cahn-Hilliard models with application to diffuse-interface tumor-growth models . International Journal for Numerical Methods in Biomedical Engineering, 30:180-203. 
[60] Xu, X., Di, Y., and Yu, H. (2018). Sharp-interface limits of a phase-field model with a generalized Navier slip boundary condition for moving contact lines. Journal of Fluid Mechanics, 849:805-833.

[61] Yang, X. and Ju, L. (2017). Linear and unconditionally energy stable schemes for the binary fluid-surfactant phase field model. Computational Methods in Applied Mechanics and Engineering, 318:1005-1029.

[62] Yu, H. and Yang, X. (2017). Numerical approximations for a phase-field moving contact line model with variable densities and viscosities. Journal of Computational Physics, (334):665-686. 